\title{
IL-10 downregulates CXCR3 expression on Th1 cells and interferes with their migration to intestinal inflammatory sites
}

\author{
M Wadwa ${ }^{1}$, R Klopfleisch ${ }^{2}$, A Adamczyk ${ }^{1}$, A Frede $^{1}$, E Pastille ${ }^{1}, K_{\text {Mahnke }}^{3}$, W Hansen ${ }^{1}$, R Geffers ${ }^{4}$, \\ KS Lang ${ }^{5}$, J Buer ${ }^{1}$, J Büning ${ }^{6,7}$ and AM Westendorf ${ }^{1,7}$
}

Inflammatory bowel disease (IBD) is characterized by chronic, uncontrolled inflammation in the intestinal mucosa. Although the etiology is poorly understood, it is widely accepted that loss of tolerance is involved in the development of IBD. Therefore, re-establishing tolerance or gut homeostasis is one of the key features in the development of new therapeutic strategies. Here we show that antigen targeting to DEC-205 on dendritic cells leads to an interleukin (IL)-10dependent downregulation of C-X-C chemokine receptor 3 (CXCR3) expression on differentiated antigen-specific T helper type 1 (Th1) cells in vivo. This downregulation interferes with the migration of Th1 cells into the gut and protects mice against severe acute and relapsing intestinal inflammation. Moreover, $\mathrm{CD} 4{ }^{+} \mathrm{CXCR} 3^{+} \mathrm{Tcells}$ are highly enriched in the inflamed mucosa of IBD patients. Interference with this pathway may therefore be a promising approach for the treatment of IBD. In conclusion, we propose a hitherto undescribed mechanism by which IL-10 can act on effector Tcells and orchestrate intestinal immune responses.

\section{INTRODUCTION}

Inflammatory bowel diseases (IBDs), including ulcerative colitis (UC) and Crohn's disease (CD), are inflammatory disorders of the gastrointestinal tract, characterized by a relapsing-remitting state of chronic inflammation. It is widely accepted that both diseases are triggered by an inappropriate immune response to antigens of commensal gut bacteria in genetically prone persons. The immunological nature of the disease arises from the observation that IBD is characterized by massive infiltration of effector $\mathrm{T}$ cells into the mucosa associated with T-cell dysfunction. This results in a loss of immunoregulatory mechanisms that maintain gut homeostasis and promotes local immune responses leading to intestinal injury. ${ }^{1}$ Current treatment options for IBD rely on the modification of immune reactions by inhibiting pro-inflammatory responses but not by correcting or augmenting aspects of the immune system that could suppress inflammation.
Despite the development of new therapies in recent decades (e.g., tumor necrosis factor blocker), a relevant proportion of patients still lack an efficient treatment. ${ }^{2,3}$ Immunosuppressant medications have significant toxic adverse effects, including myelosuppression, hepatitis, and increased risk of malignancies. Additionally, $30 \%$ of patients are primary non-responders to biological therapy and secondary failures are frequent. ${ }^{4}$ Thus, there is an urgent need to develop new, more specific therapies for IBD that aim for the re-establishment of gut homeostasis by immunoregulatory rather than immunosuppressive mechanisms.

The induction of tolerance toward specific antigens is one prominent option for the maintenance or re-establishment of gut homeostasis. ${ }^{5}$ In addition to the ability of dendritic cells (DCs) to initiate effective immune responses toward invading pathogens, these cells are also potent inducers of tolerance and, in particular, of Foxp $3^{+}$regulatory T cells (Tregs). ${ }^{6}$ In recent

\footnotetext{
${ }^{1}$ Institute of Medical Microbiology, University Hospital Essen, University of Duisburg-Essen, Essen, Germany. ${ }^{2}$ Institute of Veterinary Pathology, Freie Universität Berlin, Berlin, Germany. ${ }^{3}$ Department of Dermatology, Ruprecht-Karls University Heidelberg, Heidelberg, Germany. ${ }^{4}$ Genome Analytics, Helmholtz Centre for Infection Research, Braunschweig, Germany. ${ }^{5}$ Institute of Immunology, Medical Faculty, University of Duisburg-Essen, Essen, Germany and ${ }^{6}$ Department of Internal Medicine I, University Hospital of Schleswig-Holstein, Lübeck, Germany. Correspondence: AM Westendorf (astrid.westendorf@uk-essen.de)

${ }^{7}$ These authors contributed equally to this work. 
years, we have seen the emergence of a population of DCs that express the antigen-uptake receptor DEC-205 on their surfaces. It has been shown that targeting antigen to DEC-205 on DCs leads to highly efficient antigen presentation on major histocompatibility complex) MHC I and MHC II complexes. ${ }^{7,8}$ When this process occurs in the steady state, tolerance can be induced by several mechanisms. ${ }^{9-12}$ We and others have shown that this approach leads to the effective conversion of naive $\mathrm{CD}^{+}{ }^{+}$T cells into Foxp ${ }^{+}{ }^{+}$Tregs $^{13}$ and results in the protection in mouse models of autoimmunity, e.g., autoimmune diabetes. ${ }^{14-16}$ In addition, we have identified that, apart from the effective antigen presentation, targeting antigen to DEC-205 induces the expression of anti-inflammatory cytokines by DCs. ${ }^{17}$ This suggests that further immunomodulatory mechanisms might have a role, when antigens are targeted to DEC-205. However, it has not been addressed so far whether this approach allows the modulation of immune responses mediated by differentiated effector cells and whether it thereby protects against inflammation in the gut.

We report here that targeting antigen to DEC-205 leads to an interleukin (IL)-10-dependent downregulation of C-X-C chemokine receptor 3 (CXCR3) expression on differentiated antigen-specific $\mathrm{T}$ helper type 1 (Th1) cells in vivo. This downregulation interferes with the migration of Th1 cells into the gut and protects mice against severe intestinal inflammation. Of note, we demonstrate that $\mathrm{CD} 4{ }^{+} \mathrm{CXCR} 3{ }^{+} \mathrm{T}$ cells are highly enriched in the inflamed mucosa of IBD patients, suggesting that targeting antigen to DEC-205 could represent a potential new immunoregulatory approach for the treatment of IBD.

\section{RESULTS}

Targeting antigen to DEC-205 protects against intestinal inflammation

Various studies have shown that interferon (IFN) $-\gamma^{+}$Th1 cells act as key mediators in the onset and maintenance of IBD. ${ }^{18,19}$ Hence, we aimed to determine whether the function of Th1 cells can be controlled or blocked by targeting antigen to DEC-205. To this end, we established a new Th1-cell-mediated mouse model of intestinal inflammation. T-cell receptor (TCR) transgenic hemagglutinin (HA)-specific $\mathrm{CD} 4{ }^{+} \mathrm{T}$ cells were differentiated in vitro into IFN- $\gamma^{+}$-expressing Th1 cells (see Supplementary Figure S1 online). The adoptive transfer of differentiated HA-specific Th1 cells into VILLIN-HA transgenic mice, which specifically express HA under the control of the villin promotor active in intestinal epithelial cells, initiates a tremendous loss of body weight (Figure 1a), accompanied by massive destruction of the intestinal architecture of both the small intestine and colon (Figure 1b), and expands the numbers of $\mathrm{HA}$-specific $\mathrm{CD}^{+}{ }^{+} \mathrm{T}$ cells in the mesenteric lymph nodes (MLNs) and lamina propria (LP) of the colon 5 days after transfer (Figure 1c).

To analyze the effect of targeting antigen to DEC-205 on antigen-specific Th1 cells and the related intestinal inflammation, we chemically coupled the $\mathrm{HA}_{110-120}$ peptide to an $\alpha$-DEC205 antibody (DEC-HA) or to a non-binding isotype control antibody (ISO-HA). We injected DEC-HA, ISO-HA, or phosphate-buffered saline (PBS) intraperitoneally (i.p.) into VILLIN-HA transgenic mice on days -2 and -1 before the adoptive transfer of HA-specific Th1 cells (Figure 1d). Remarkably, VILLIN-HA transgenic mice treated with DEC-HA lost significantly less body weight within 5 days than did control mice (Figure 1e). In line with this finding, histopathological analysis detected strongly less severe inflammation in the small intestine and colon of mice treated with DEC-HA than in their control counterparts (Figure 1f).

In previous studies, we have shown that targeting antigen to DEC-205 in vivo leads to the de novo generation and expansion of antigen-specific Foxp $3^{+}$Tregs. ${ }^{14,17}$ Interestingly, the percentage and absolute number of Foxp ${ }^{+}$Tregs in all the analyzed compartments did not differ significantly between the groups, neither when gated on transferred HA-specific CD4 ${ }^{+}$ $\mathrm{T}$ cells (Figure 2a) nor when gated on endogenous $\mathrm{CD} 4{ }^{+}$ $\mathrm{T}$ cells (data not shown). However, we observed significantly lower frequencies and total numbers of adoptively transferred Th1 cells in the MLNs and colons of mice treated with DEC-HA than in the colons of control mice (Figure 2b). No differences were detected in the non-gut-associated lymphoid tissues. Of note, we determined an increase in the frequencies and numbers of Th1 cells in the blood of mice treated with DEC-HA compared with control mice. We excluded the possibility that the proliferative capacity of HA-specific Th1 cells was altered by DEC-HA treatment (data not shown). These results suggest a DEC-HA-mediated effect on the migration of effector cells into the gut.

\section{DEC-HA treatment modulates the migration of Th1 cells}

To gain insights into the phenotype and function of Th1 cells after treatment with DEC-HA, we analyzed the characteristics of these cells by global gene expression profiling. We adoptively transferred HA-specific Th1 cells into VILLIN-HA transgenic mice that had been treated with DEC-HA, ISO-HA, or PBS. Five days after transfer, we re-isolated $\mathrm{HA}$-specific $\mathrm{CD} 4{ }^{+}$ $\mathrm{T}$ cells from the colonic LP and subjected them to microarray analysis. A general overview on the changes in expression levels after PBS, ISO-HA, and DEC-HA treatment is visualized by volcano plots (see Supplementary Figure S2). Because of the lower frequency of HA-specific Th1 cells in the intestine and the higher frequency in the blood of VILLIN-HA transgenic mice treated with DEC-HA, we first addressed the question whether the migration of Th1 cells into the colon was altered. Interestingly, most of the common migration markers, including the gut-homing markers CCR9 and integrin $\alpha_{4} \beta_{7}$, were not differentially expressed or were only slightly downregulated after treatment with DEC-HA. However, the expression of the chemokine receptors CXCR3 and CXCR5 on HA-specific Th1 cells was exclusively downregulated after treatment with DEC-HA (Figure 3a).

To elucidate the potential relevance of these receptors in the present inflammation model, we measured the expression of the respective chemokine receptor ligands (CXCL9 and CXCL10 for CXCR3; CXCL13 for CXCR5) in the gut of 
a

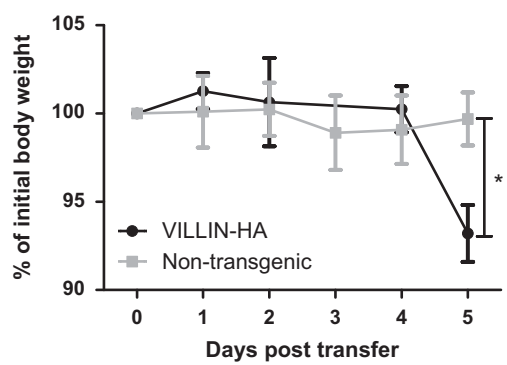

C

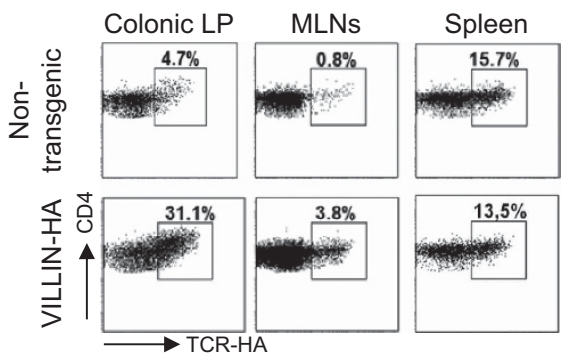

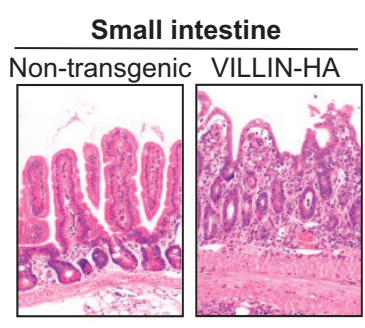

Colon

Non-transgenic VILLIN-HA

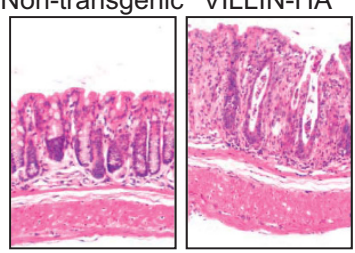

d

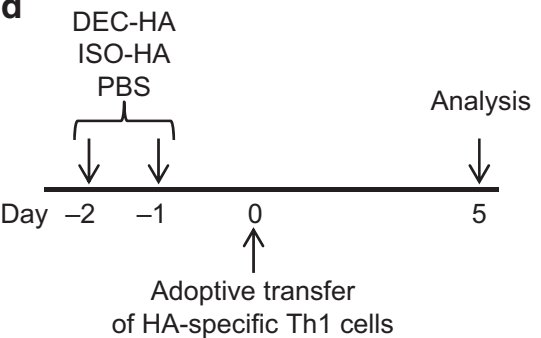

e

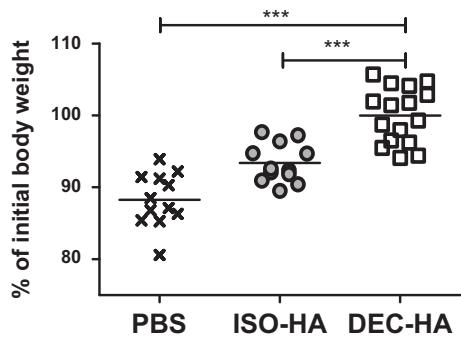

f
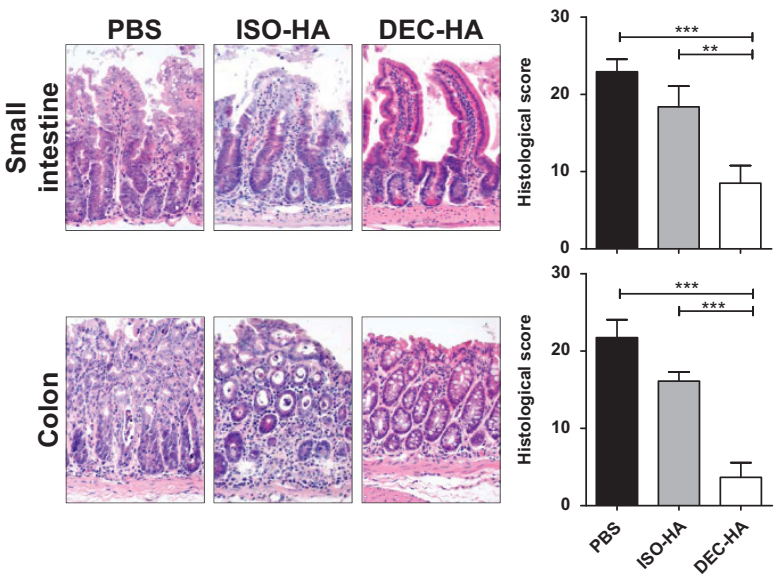

Figure 1 Targeting antigen to DEC-205 protects against Thelper type 1 (Th1)-cell-mediated intestinal inflammation. Hemagglutinin (HA)-specific Th1 cells were adoptively transferred into VILLIN-HA transgenic mice or non-transgenic littermates. (a) Mice were monitored daily for clinical signs of sickness and loss of body weight. (b) On day 5 after transfer, histopathological analysis was performed on sections from the small intestine and colon. (c) Frequencies of HA-specific (TCR-HA) CD4 ${ }^{+} \mathrm{T}$ cells in the colonic lamina propria (LP), the mesenteric lymph nodes (MLNs), and the spleen were determined by flow cytometry. (d) VILLIN-HA transgenic mice were injected intraperitoneally with DEC-HA, ISO-HA, or sterile phosphate-buffered saline (PBS) on days -2 and -1 before adoptive transfer of HA-specific Th1 cells. Mice were monitored daily for clinical signs of sickness. On day 5 after transfer, (e) the body weight of the mice was determined, and (f) histopathological analysis was performed on sections of the small intestine and colon. Data from four independent experiments are shown as means \pm s.e.m. Statistical analysis was performed using Student's $t$-test (a) or one-way analysis of variance $(\mathbf{e}+\mathbf{f})\left({ }^{\star} P<0.05 ;{ }^{* \star} P<0.01 ;{ }^{* \star \star} P<0.001\right)$.

VILLIN-HA transgenic mice and their non-transgenic littermates after the transfer of HA-specific Th1 cells. The expression of CXCL9 and CXCL10 was tremendously upregulated in diseased VILLIN-HA transgenic mice, whereas the expression of CXCL13 in the gut of diseased mice was not different from that of healthy control mice (Figure 3b). Hence, we decided to focus further on the function of CXCR3 and its ligands CXCL9 and CXCL10 in this model and confirmed the 
a
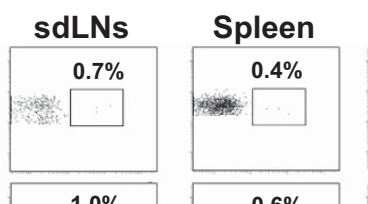

Blood
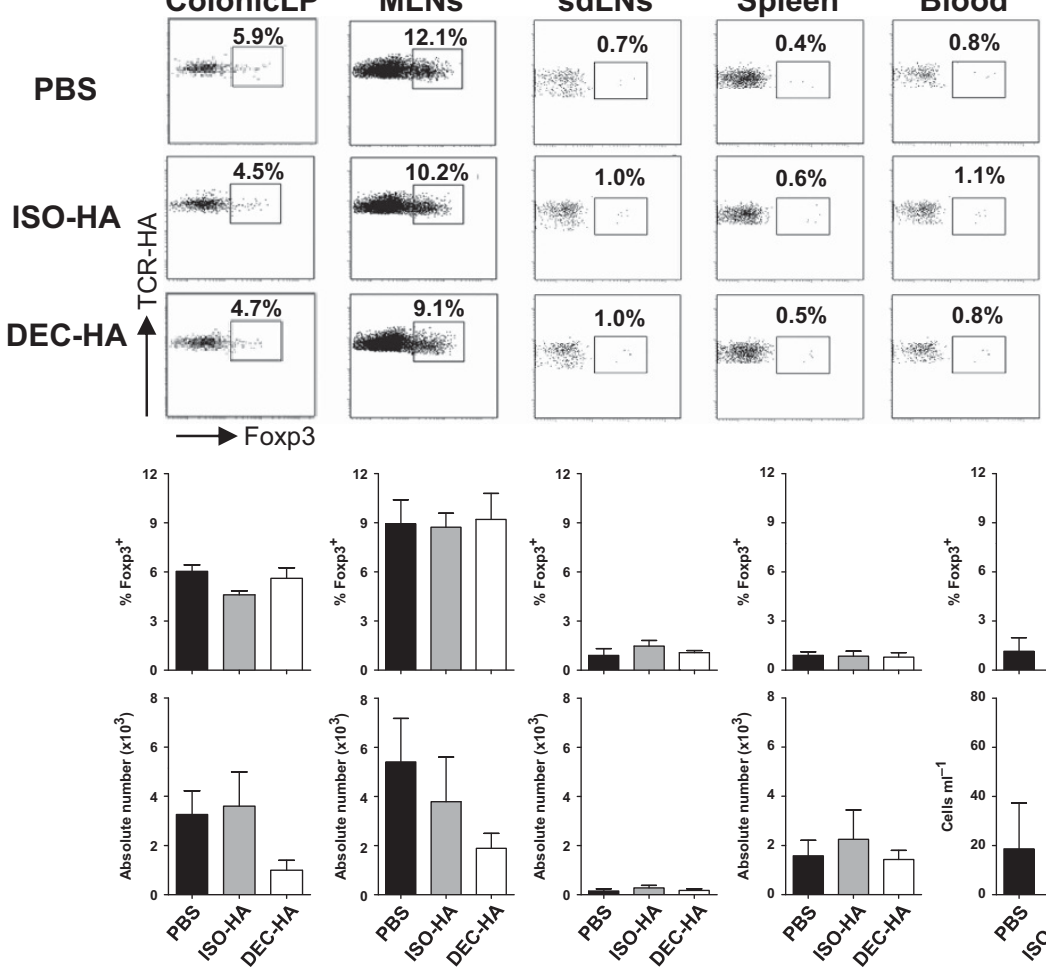

b
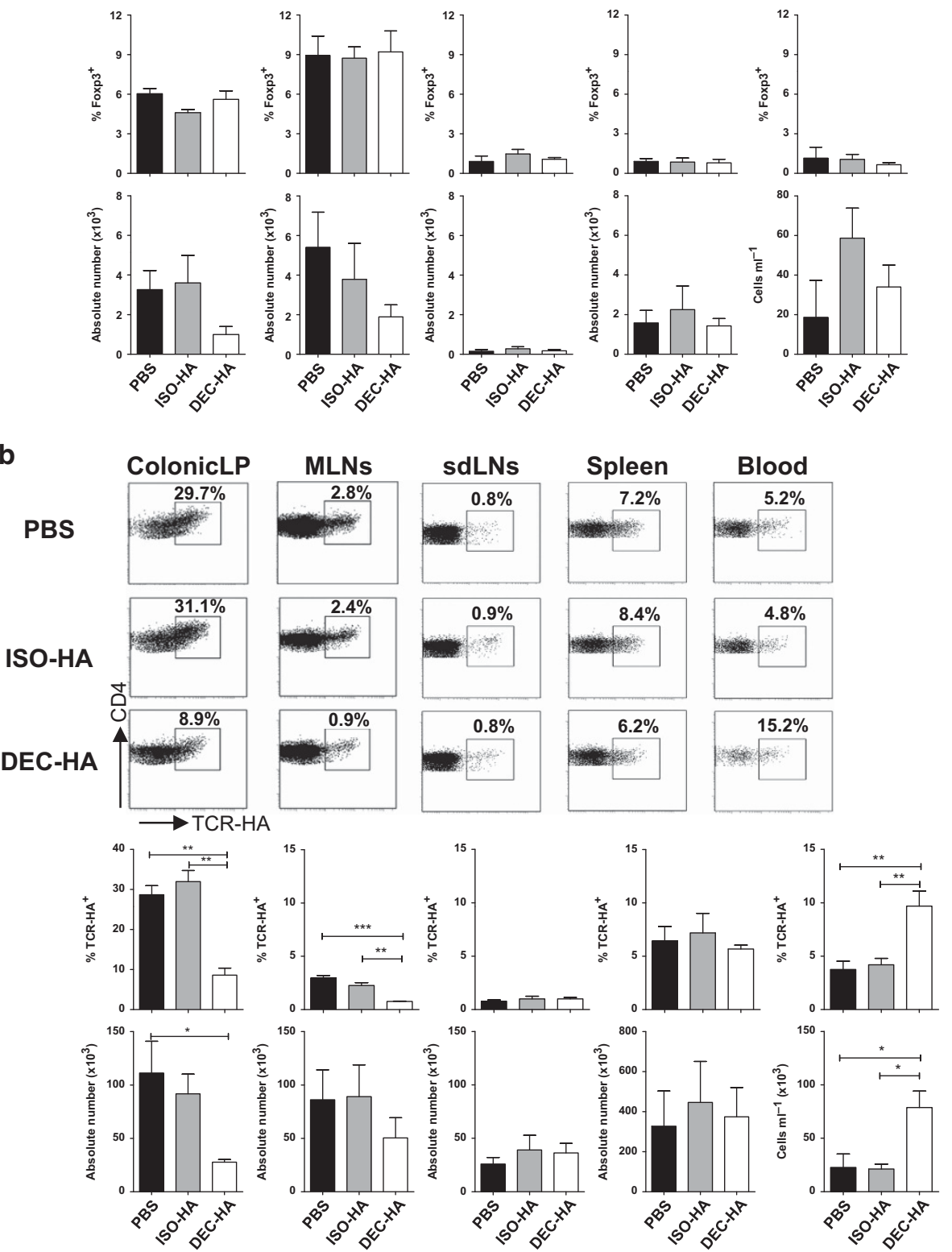

Figure 2 Frequencies and total numbers of hemagglutinin (HA)-specific T helper type 1 (Th1) cells are diminished in the gut of VILLIN-HA transgenic mice after targeting antigen to DEC-205. HA-specific Th1 cells were adoptively transferred into DEC-HA, ISO-HA, or phosphate-buffered saline (PBS)-treated VILLIN-HA transgenic mice. Five days after transfer, lymphocytes from the colonic lamina propria (LP), mesenteric lymph nodes (MLNs), skin-draining lymph nodes (sdLNs), spleen and blood were counted and analyzed by flow cytometry. (a) Adoptively transferred HA-specific (TCR-HA) $\mathrm{CD}^{+}{ }^{+} \mathrm{T}$ cells were gated and analyzed for the expression of Foxp3. (b) All CD4 ${ }^{+} \mathrm{T}$ cells were gated, and numbers and frequencies of HA-specific (TCR-HA) T cells were determined. Data from two independent experiments are shown as means \pm s.e.m. Statistical analysis was performed with one-way analysis of variance $\left({ }^{\star} P<0.05,{ }^{* *} P<0.01 ;{ }^{* * *} P<0.001\right)$. 

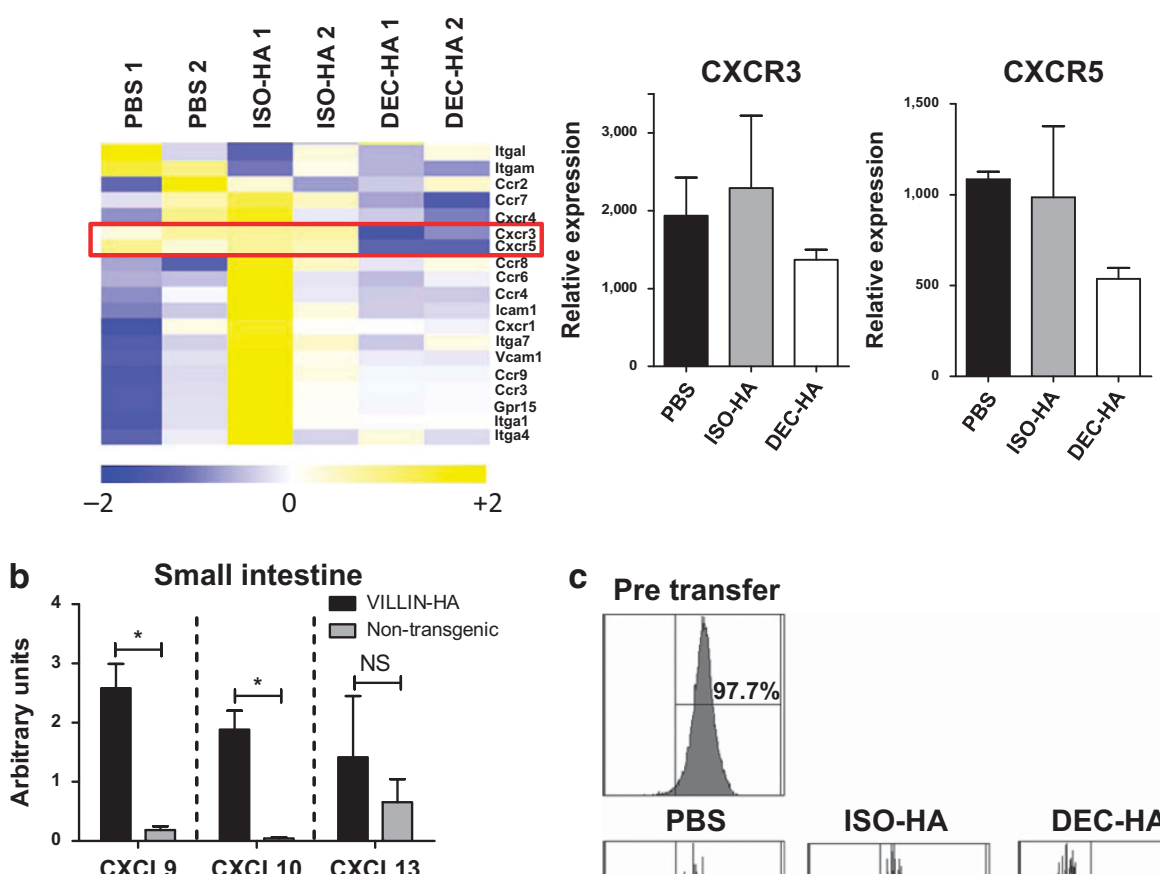

C Pre transfer
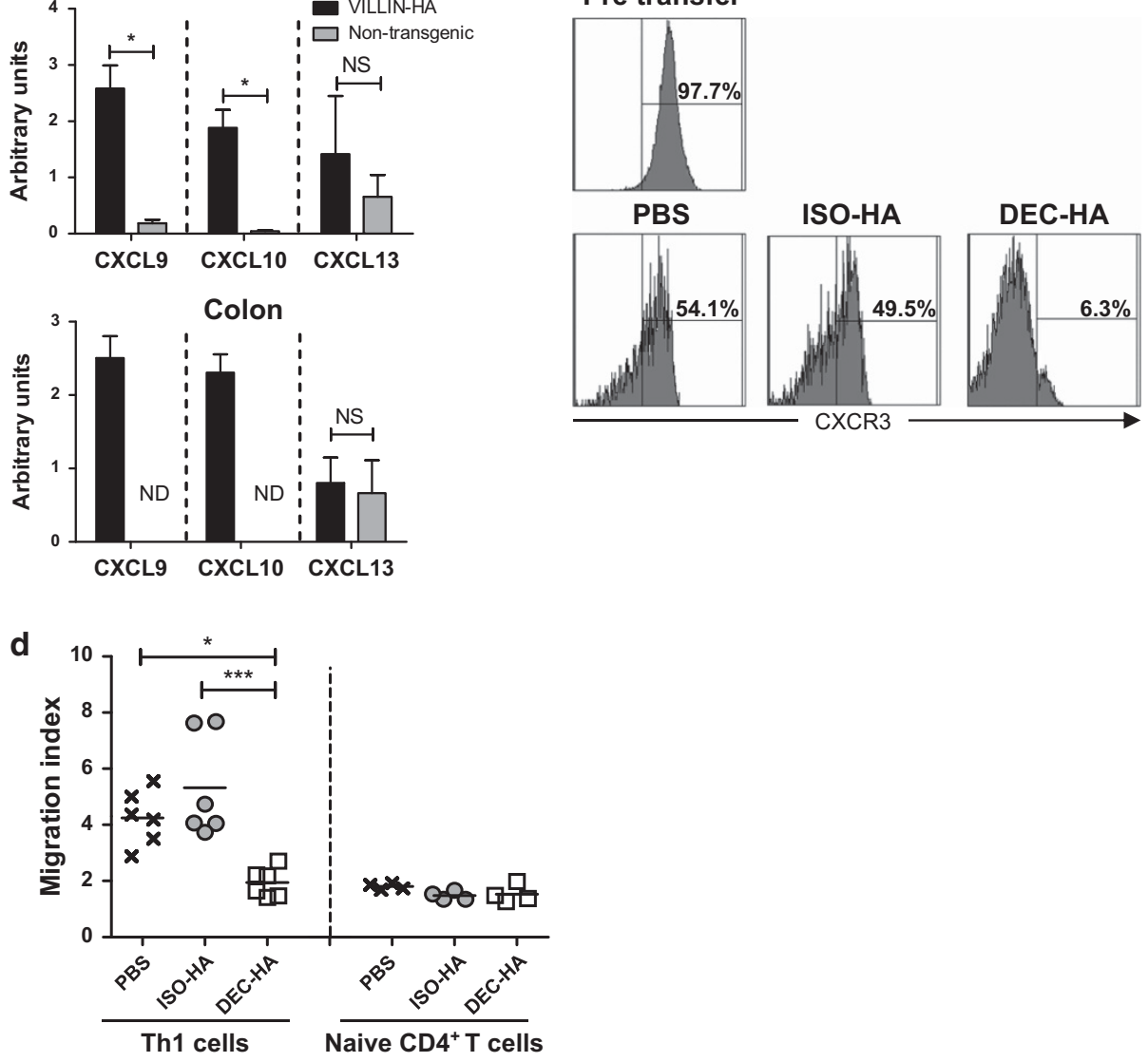

Figure 3 Treatment with DEC-HA leads to downregulation of C-X-C chemokine receptor 3 (CXCR3) on T helper type 1 (Th1) cells. VILLIN-HA transgenic mice were injected intraperitoneally (i.p.) with DEC-HA, ISO-HA, or phosphate-buffered saline (PBS) before adoptive transfer of HA-specific Th1 cells. Five days after cell transfer, HA-specific $\mathrm{CD} 4{ }^{+} \mathrm{T}$ cells were re-isolated from the colonic lamina propria (LP) by flow cytometry and subjected to gene expression profiling. (a) The heat map depicts the expression of selected genes related to cell migration. Relative expression of CXCR3 and CXCR5 is shown individually. (b) The expression of the CXCR3 ligands CXCL9 and CXCL10 and of the CXCR5 ligand CXCL13 was determined by quantitative real-time PCR in biopsy samples from the small intestines and colons of Th1-cell-transferred VILLIN-HA transgenic mice and non-transgenic littermates. VILLIN-HA transgenic mice were injected i.p. with DEC-HA, ISO-HA, or PBS, followed by the adoptive transfer of HA-specific Th1 cells. (c) Five days later, $\mathrm{HA}$-specific CD4 ${ }^{+} \mathrm{T}$ cells from the colonic LP were analyzed for CXCR3 expression by flow cytometry. HA-specific Th1 cells were adoptively transferred into BALB/c mice treated with DEC-HA, ISO-HA, or PBS and were re-isolated from the spleens by flow cytometry 7 days after transfer. (d) T cells were used in in vitro transwell migration assays with CXCL10 as chemoattractant. Naive HA-specific CD4 ${ }^{+}$cells served as a negative control. Data from three independent experiments are shown as means \pm s.e.m. Statistical analysis was performed with Student's $t$-test (b) or one-way analysis of variance (d) $\left({ }^{\star} P<0.05 ;{ }^{* *} P<0.001 ; \mathrm{NS}\right.$, not significant; ND, not detectable).

results of the microarray for CXCR3 expression on protein level by flow cytometry (Figure 3c). To determine whether the decrease in CXCR3 expression by Th1 cells had functional implications on the migration behavior of these cells, we transferred HA-specific Th1 cells into BALB/c mice that had been treated with DEC-HA, ISO-HA, or PBS. We re-isolated 
these cells from the spleens on day 7 after transfer and used them in an in vitro transwell migration assay with recombinant CXCL10 as a chemoattractant. Naive HA-specific CD ${ }^{+}$T cells, which only slightly express CXCR3, served as negative controls. HA-specific Th1 cells isolated from mice treated with PBS or ISO-HA strongly migrated through the transwell in response to CXCL10, whereas treatment with DEC-HA completely abolished the migration of these cells toward CXCL10; the results were comparable to those obtained with the naive $\mathrm{CD}^{+}{ }^{+} \mathrm{T}$ cells (Figure 3d). To verify that the downregulation of CXCR3 and the reduced migration after antigen targeting to DEC-205 was not restricted to the HA model antigen but rather a general phenomenon, we repeated the experiments with $\alpha$-DEC-205 monoclonal antibody coupled to an MHC class II restricted ovalbumin (OVA) peptide (DEC-OVA) and used OVA-specific Th1 polarized cells. In line with the former results, treatment of mice with DEC-OVA leads to a downregulation of CXCR3 expression on OVA-specific Th1 cells (see Supplementary Figure S3a) and abolished the in vitro migration toward recombinant CXCL10 (see Supplementary Figure S3b).

\section{Differential expression of CXCR3 in IBD patients}

To determine a potential role of CXCR3-expressing CD4 ${ }^{+}$ $\mathrm{T}$ cells during IBD in more detail, we analyzed the presence of $\mathrm{CD}^{+}{ }^{+} \mathrm{CXCR}^{+}{ }^{+} \mathrm{T}$ cells in peripheral blood mononuclear cells (PBMCs) and biopsy samples from the gut of healthy volunteers, patients with IBD in remission, and those with active CD or UC. Interestingly, the percentage of CXCR3expressing $\mathrm{CD}^{+}{ }^{+} \mathrm{T}$ cells was significantly lower in the blood of patients with active $\mathrm{CD}$ and $\mathrm{UC}$, and a gradual decrease in the percentage of these cells was seen from healthy volunteers to patients with disease in remission to those with active disease (Figure 4a).

In contrast, determining the number of $\mathrm{CD} 4{ }^{+} \mathrm{CXCR} 3^{+}$ $\mathrm{T}$ cells in the gut mucosa led to reciprocal results. In healthy volunteers and in IBD patients in remission, $\mathrm{CD} 4{ }^{+} \mathrm{CXCR} 3^{+}$ $\mathrm{T}$ cells were only barely detectable in the colon and ileum, but the numbers of these cells were significantly higher during active $\mathrm{CD}$ or UC (Figure $4 \mathbf{b}$ ). These findings suggest that the migration of $\mathrm{CD}^{+}{ }^{+} \mathrm{CXCR} 3^{+}$effector $\mathrm{T}$ cells to the site of inflammation is elevated during active IBD and also imply that the specific blockade or downregulation of CXCR3 may be an efficient therapeutic strategy for the treatment of IBD.

\section{A CXCR3 antagonist ameliorates Th1-cell-induced intestinal inflammation}

To compare the efficiency of systemic CXCR3 blocking and DEC-205-mediated downregulation of CXCR3 expression on antigen-specific Th1 effector cells for the prevention of intestinal inflammation, we treated VILLIN-HA transgenic mice either with DEC-HA and PBS as previously described or with a CXCR3 antagonist administered day to day (Figure 5a). On day 5 after the adoptive transfer of HA-specific Th1 cells, PBS-treated mice lost as much as $10 \%$ of their initial body weight. In contrast, the body weight of mice treated with DEC-HA or with CXCR3 antagonist remained constant
(Figure 5b). In addition, histological analysis demonstrated that the inflammation status in the small intestine and the colon of CXCR3 antagonist-treated mice was lower than that in the colon and small intestine of PBS-treated animals (Figure 5c). Remarkably, nearly no pathological response was detectable in DEC-HA-treated animals. In line with these findings, the frequencies of transferred HA-specific Th1 cells were significantly lower in the LP and MLNs of mice that had been treated with the CXCR3 antagonist or DEC-HA than in control animals injected with PBS (Figure 5d). In summary, these findings indicate that the systemic blocking of CXCR3 by an antagonist hinders the migration of effector $\mathrm{T}$ cells to the gut and is sufficient to reduce intestinal inflammation. However, the particular downregulation of the expression of CXCR3 by antigen-specific Th1 cells brought about by antigen targeting to DEC-205 seems to be more effective in the prevention of gut inflammation than treatment with a CXCR3 antagonist.

\section{The protective activity of targeting antigen to DEC-205 is IL- 10 dependent}

We have previously shown that targeting antigen to the DEC-205 receptor on DCs with single-chain fragments leads not only to enhanced antigen presentation but also to increased secretion of the anti-inflammatory cytokine IL-10. ${ }^{17}$ To determine whether elevated IL-10 production is involved in the downregulation of CXCR3 on Th1 cells, we made use of $\mathrm{CD} 11 \mathrm{c}-\mathrm{cre} \times \mathrm{IL}-10^{\text {flox/flox }}$ mice that specifically lack IL-10 expression in $\mathrm{CD} 11 \mathrm{c}^{+}$DCs. CD11c-cre $\times \mathrm{IL}-10^{\text {flox/flox }}$ or IL-10 flox/flox control mice were injected with DEC-HA, ISO-HA, or PBS. One day later, sera and additionally CD11c ${ }^{+}$ DCs from the spleens of mice were analyzed for secretion of IL-10. Indeed, we found that IL-10 was detectable in the sera of IL-10 flox/flox DEC-HA but not in control-treated mice. Moreover, ex vivo isolated splenic DCs from mice injected with DEC-HA produced significantly more IL-10 than did DCs isolated from mice treated with ISO-HA or from PBS-treated control mice. Strikingly, IL-10 expression was completely abolished when mice were deficient in functional IL-10 expression in $\mathrm{CD}_{11 \mathrm{c}^{+}}$DCs (Figure 6a). Further comprehensive cytokine analysis of DCs isolated from the colonic, MLNs, and spleens revealed that IL-10 and transforming growth factor (TGF)- $\beta$ expression was upregulated in the spleens and MLNs but not in the colonic lamina of DEC-HA treated mice (see Supplementary Figure S4a). However, DEC-HA treatment had no effect on the expression of pro-inflammatory cytokines such as IL-1 $\beta$, IL- 6 , tumor necrosis factor- $\alpha$, and the expression of activation markers CD40, CD80, CD86, or MHC-II (see Supplementary Figure S4b).

To determine whether DEC-205-mediated upregulation of IL-10 by DCs influences the migration activity of Th1 cells, CD11c-cre $\times$ IL10 flox/flox or IL10 flox/flox control mice were injected i.p. with DEC-HA or ISO-HA prior to adoptive transfer of HA-specific Th1 cells. Six days after transfer, CXCR3 expression was analyzed on transferred Th1 cells ex vivo by flow cytometry. In IL-10 $0^{\text {flox/flox }}$ control mice, CXCR3 expression was significantly decreased on Th1 cells after DEC-HA treatment. 

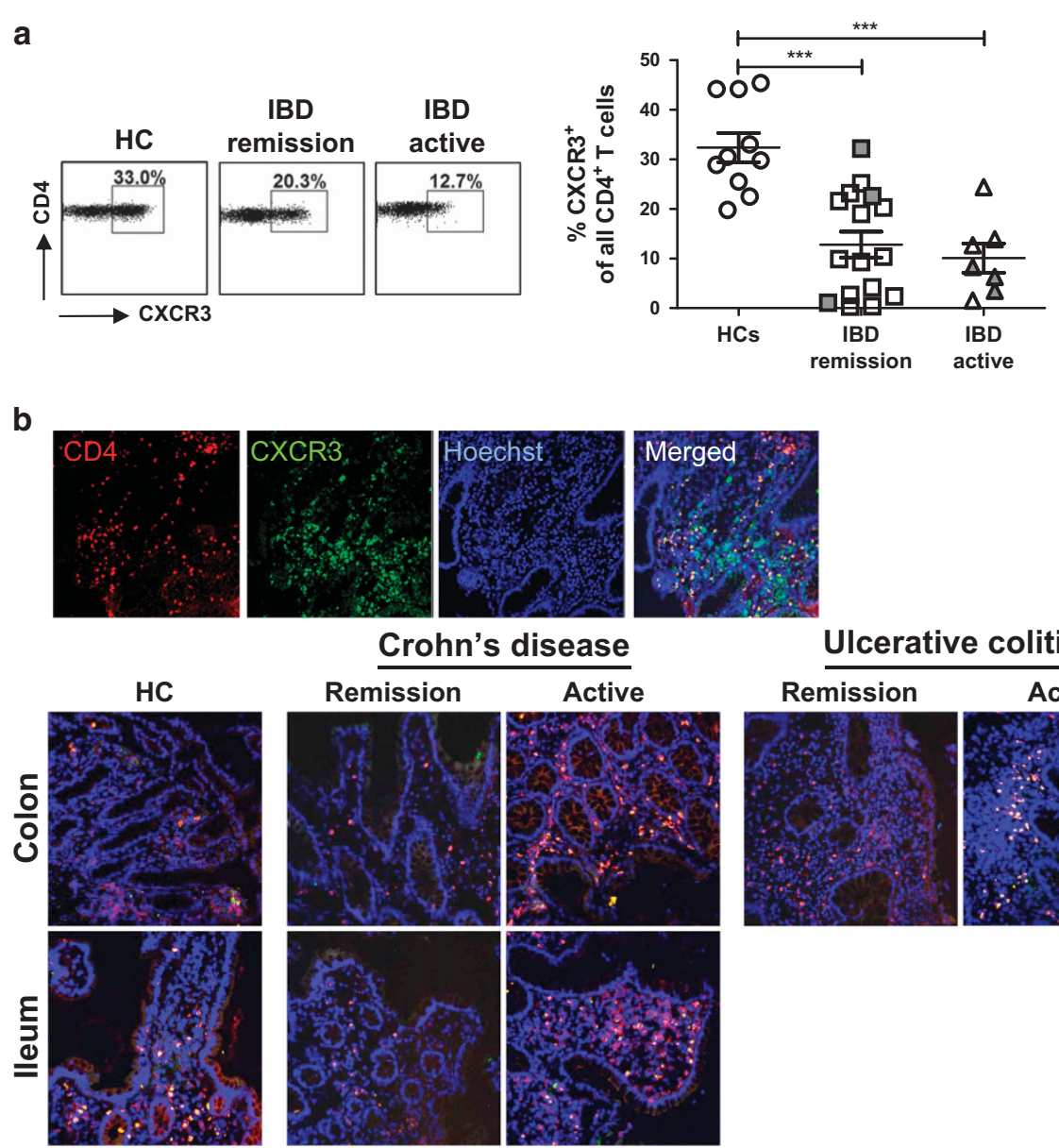

Active

\section{Ulcerative colitis}
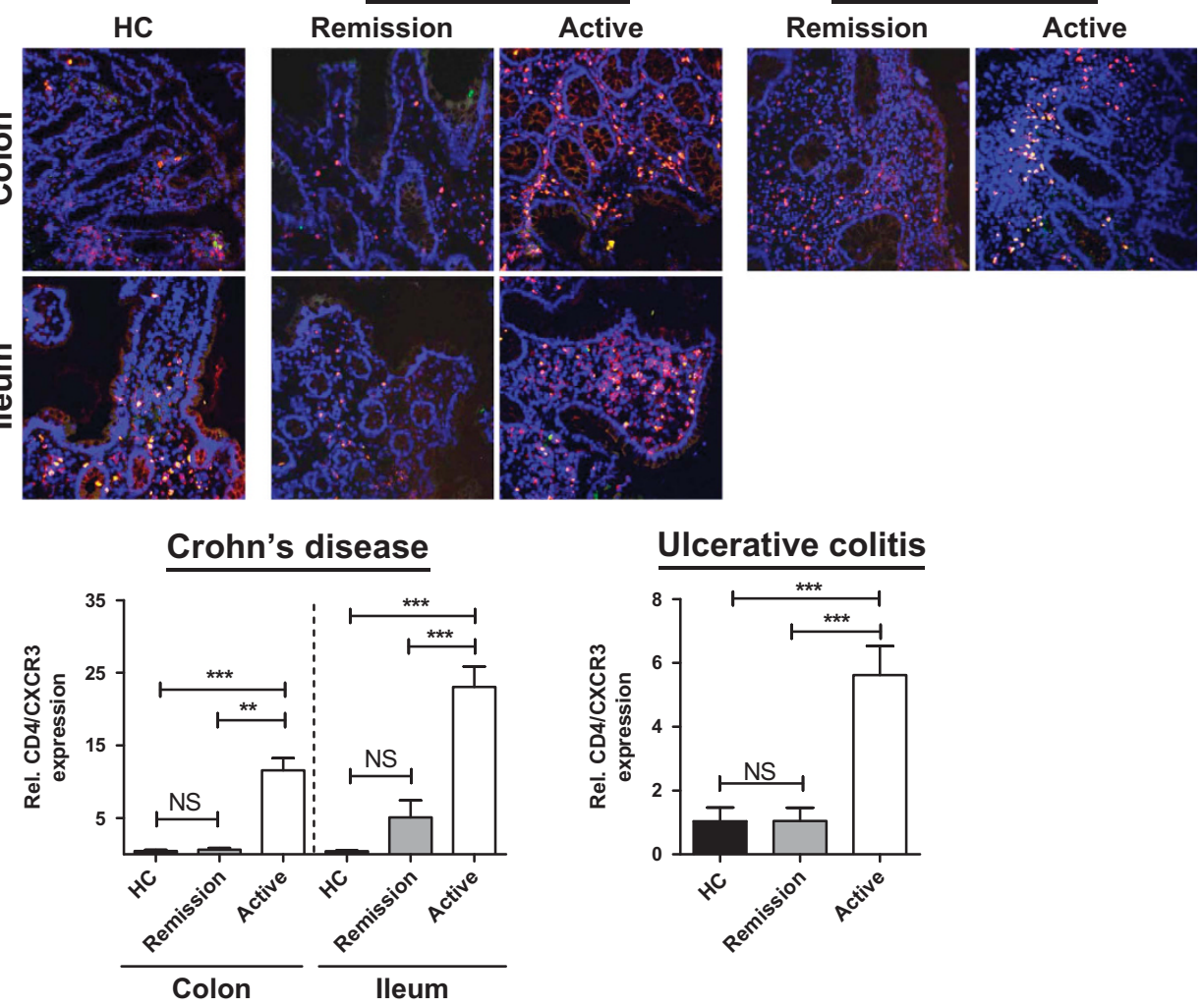

Figure $4 \mathrm{CD}^{+}{ }^{+} \mathrm{CXCR}^{+} \mathrm{T}$ cells are decreased in the periphery and increased in the inflamed intestinal mucosa of patients with inflammatory bowel disease (IBD). (a) Peripheral blood mononuclear cells from healthy control subjects (HCs) and from IBD patients in remission or with active disease were analyzed for the presence of $\mathrm{CD} 4{ }^{+} \mathrm{CXCR}^{+} \mathrm{T}$ cells by flow cytometry. Representative dot plots are shown. Data are summarized in the scatter plot. Grey symbols, data from patients with Crohn's Disease; white symbols, data from patients with ulcerative colitis. (b) Cryo-sections of colonic and ileal biopsy samples from HCs, patients with Crohn's Disease, and patients with ulcerative colitis in remission or with active inflammatory relapse were fluorescently labelled with antibodies against CD4 and C-X-C chemokine receptor 3 (CXCR3). Hoechst dye was used to label cell nuclei. Representative images from the colon and ileum of each analyzed group are shown. For quantification of $\mathrm{CD} 4{ }^{+} \mathrm{CXCR} 3^{+}$cells, the number of blue pixels (cell nuclei) was divided by the number of yellow pixels (CD4 ${ }^{+} \mathrm{CXCR}^{+}$) in each image and is depicted as relative CD4/CXCR3 expression in the bar diagrams. Data are summarized as means \pm s.e.m. Statistical analysis was performed with one-way analysis of variance $\left({ }^{\star \star} P<0.01\right.$; ${ }^{* \star *} P<0.001$; NS, not significant).

Strikingly, CXCR3 expression remained high on Th1 cells re-isolated from CD11c-cre $\times$ IL10 $0^{\text {flox/flox }}$ mice despite DEC-HA treatment (Figure 6b). To further analyze the migratory properties of Th1 cells after DEC-HA and ISO-HA treatment, transferred Th1 cells were re-sorted from the spleens of these mice and subjected to in vitro migration assays with CXCL10 as a chemoattractant. Consistent with the finding that CXCR3 expression of the transferred Th1 cells was not altered in CD11c-cre $\times$ IL10 flox/flox mice, also the in vitro migration of these cells remained equally strong. In contrast, Th1 cells 
a

a)

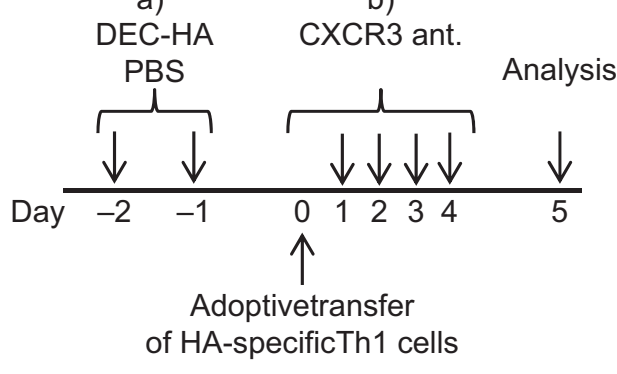

b

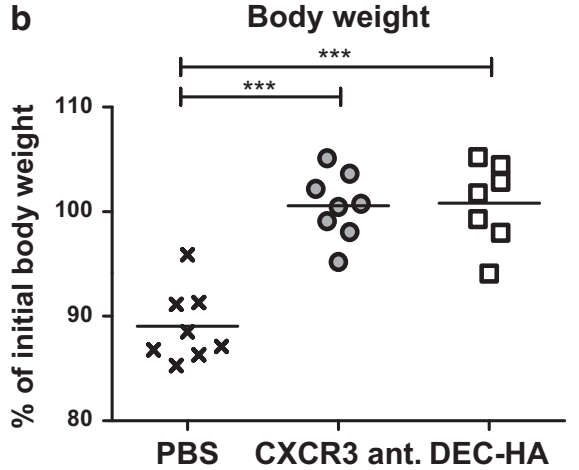

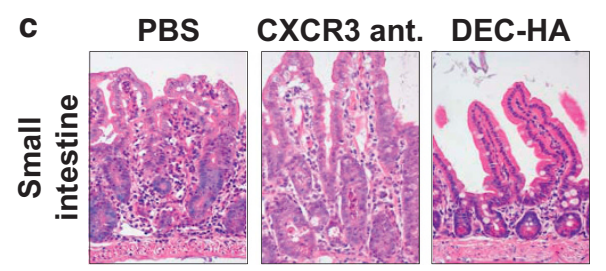
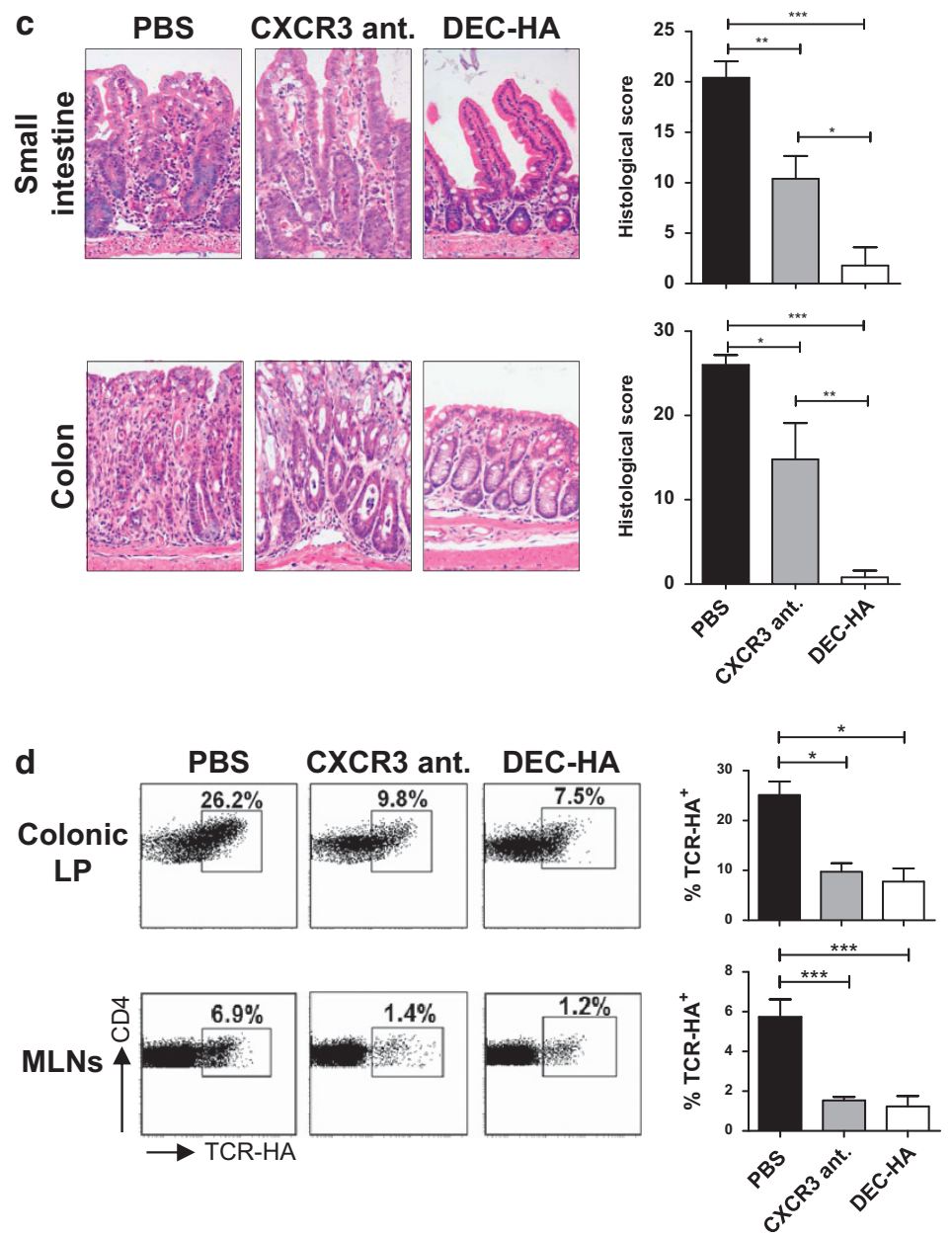

Figure 5 Treatment of VILLIN-HA mice with a C-X-C chemokine receptor 3 (CXCR3) antagonist leads to protection against intestinal inflammation. (a) HA-specific Thelper type 1 (Th1) cells were adoptively transferred into VILLIN-HA transgenic mice treated with DEC-HA or phosphate-buffered saline (PBS). One group of mice was given daily intraperitoneal (i.p.) injections of $150 \mu \mathrm{g}$ CXCR3 antagonist (CXCR3 ant.) until day 4 after transfer. (b) Body weight and clinical signs of sickness were monitored. (c) On day 5 after transfer, mice were subjected to histopathological analysis. (d) Frequencies of $\mathrm{HA}$-specific $\mathrm{CD}^{+}{ }^{+} \mathrm{T}$ cells in the colonic lamina propria (LP) and the mesenteric lymph nodes (MLNs) of the mice were determined by flow cytometry. Data from three independent experiments are shown as means \pm s.e.m. Statistical analysis was performed with one-way analysis of variance $\left({ }^{\star} P<0.05\right.$; ${ }^{\star *} P<0.01 ;{ }^{* *} P<0.001 ;$ NS, not significant).

isolated from DEC-HA-treated IL10 flox/flox control mice showed significantly lower migration toward CXCL10 in vitro (Figure 6c).

Next we examined whether treatment with a neutralizing $\alpha$-IL-10 antibody could abolish DEC-HA-mediated protection in vivo. VILLIN-HA transgenic mice were treated with
DEC-HA or ISO-HA in the presence of a neutralizing $\alpha$-IL-10 or an isotype control antibody before the adoptive transfer of HA-specific Th1 cells (Figure 7a). Strikingly, on day 5 after cell transfer the protective effect of DEC-HA was abolished when $\alpha$-IL-10 was co-administered. Mice exhibited a reversed loss of body weight (Figure $\mathbf{7 b}$ ) and a more severe intestinal 

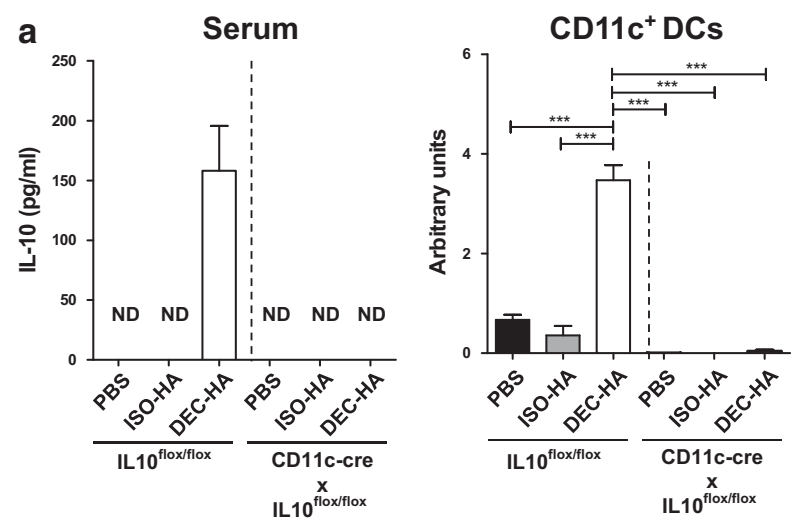

b
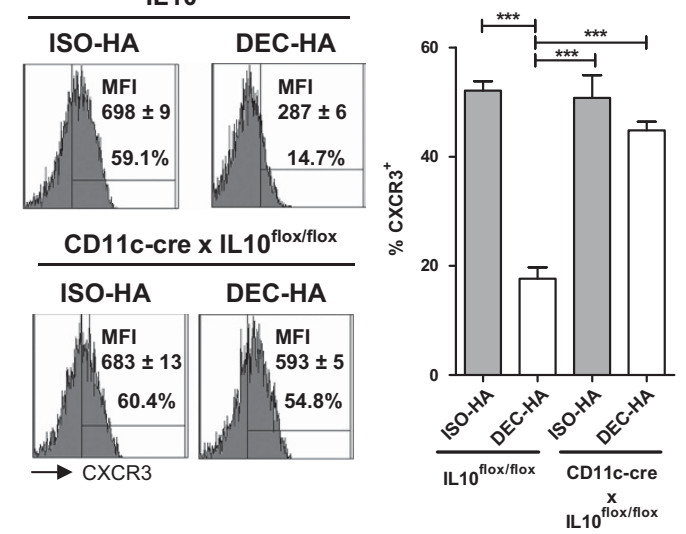

C

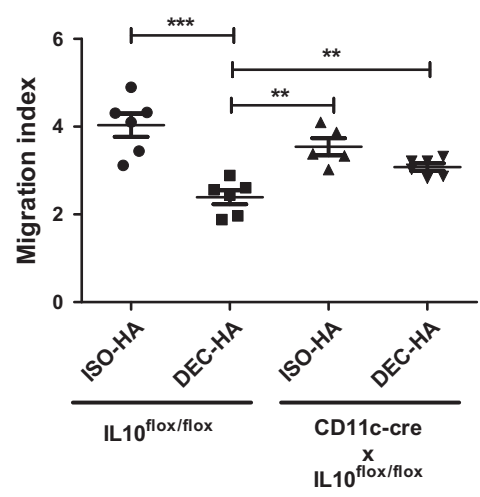

Figure 6 In vitro migration of hemagglutinin (HA)-specific Thelper type 1 (Th1) cells is increased in the absence of interleukin (IL)-10. IL10 flox/flox or CD11c-cre $\times$ IL10 flow/flox mice were injected intraperitoneally (i.p.) with DEC-HA, ISO-HA, or phosphate-buffered saline (PBS). (a) Twenty-four hours later, serum of the mice was collected and analyzed for IL-10 with Luminex technology. Additionally, $\mathrm{CD}_{11 \mathrm{c}^{+}}$dendritic cells (DCs) were isolated from the spleens and analyzed ex vivo for IL-10 expression by quantitative real-time PCR. IL-10 flox/flox or CD11c-cre $\times I L 10^{\text {flox/flox }}$ mice were injected i.p. with ISO-HA or DEC-HA followed by the transfer of HAspecific Th1 cells. (b) On day 4 after transfer, Th1 cells were analyzed ex vivo for the expression of C-X-C chemokine receptor 3 by flow cytometry. (c) HA-specific Th1 cells were re-isolated from the spleens by flow cytometry and used in in vitro transwell migration assays with C-X-C chemokine ligand 10 as chemoattractant. Data from two independent experiments are shown as means \pm s.e.m. Statistical analysis was performed with one-way analysis of variance $\left({ }^{\star \star} P<0.01 ;{ }^{\star \star \star} P<0.001\right.$; $\mathrm{ND}$, not detectable). MFI, mean fluorescence intensity. inflammation (Figure 7c). Moreover, the frequencies of HA-specific Th1 cells and CXCR3 surface expression in the colon and MLNs were higher than in mice treated with DEC-HA alone (Figure 7d,e). Of note, neither treatment with a DEC-205 monoclonal antibody without antigen nor treatment with an antibody coupled to an irrelevant antigen was sufficient to protect against intestinal inflammation (see Supplementary Figure S5). These findings clearly suggest a novel mechanism by which IL-10 can act on effector T cells, thereby mediating its anti-inflammatory properties.

To further elucidate the mechanism of how IL-10 leads to downregulation of CXCR3 on Th1 cells, we first analyzed whether Th1 cells express the IL-10 receptor (IL-10R) and could therefore respond to IL-10 directly. We included in vitro polarized antigen-specific Th 1 cells, naive $\mathrm{CD} 4{ }^{+} \mathrm{T}$ cells, and Tregs, known to express high levels of IL-10R.$^{20}$ We detected only a weak but existing expression of IL-10R on Th1 cell (see Supplementary Figure S6a). To check whether the direct binding of IL-10 influences CXCR3 expression on Th1 cells, polarized Th1 cells were re-stimulated in the presence or absence of recombinant mouse IL-10 or a neutralizing $\alpha$-IL-10 antibody, and the expression of CXCR3 was determined. Importantly, no differences in the expression of CXCR3 were detected (see Supplementary Figure S6b) suggesting an indirect effect of IL-10 on CXCR3 expression on Th1 cells.

DEC-HA treatment protects against inflammatory relapse IBD is characterized by inflammatory relapses and inflammation-free remission phases. Because of the existing in vitro and in vivo data, we cannot exclude that targeting antigen to DEC-205 under active inflammation would further aggravate an inflammatory response. Therefore, we analyzed whether DEC-HA treatment of VILLIN-HA mice on days 1 and 2 after the adoptive transfer of HA-specific Th1 cells alters the inflammatory response (Figure 8a). Interestingly, this procedure resulted in an intermediate state of inflammation. VILLIN-HA transgenic mice treated with DEC-HA after transfer lost less body weight within 5 days than did control mice (Figure 8b). In line with this finding, histopathological analysis detected less severe inflammation in the small intestine and colon of mice treated with DEC-HA than in their control counterparts (Figure 8c). However, pretreatment of VILLIN-HA mice with DEC-HA before adoptive transfer of HA-specific Th1 cells (DEC-HA control) is much more effective in the prevention of gut inflammation than posttreatment with DEC-HA. Therefore, we analyzed whether DEC-HA treatment of VILLIN-HA mice during an inflammation-free remission phase would be a suitable therapeutic option. To this end, low numbers of HA-specific Th1 cells were adoptively transferred into VILLIN-HA mice to initiate a non-lethal inflammation of the gut (Figure 8d). The inflammatory response was monitored by the loss of body weight and histology of the small intestine and colon at day 6 after transfer. After 3 weeks, the mice had completely recovered from intestinal inflammation, closely resembling the situation in the inflammation-free remission phases of IBD patients (Figure 8e,f). On days 33 and 34 after the 
a

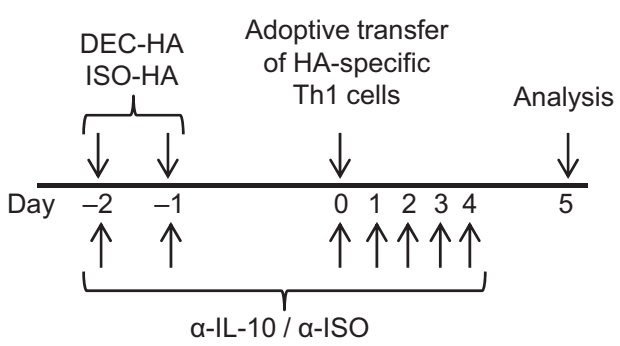

c
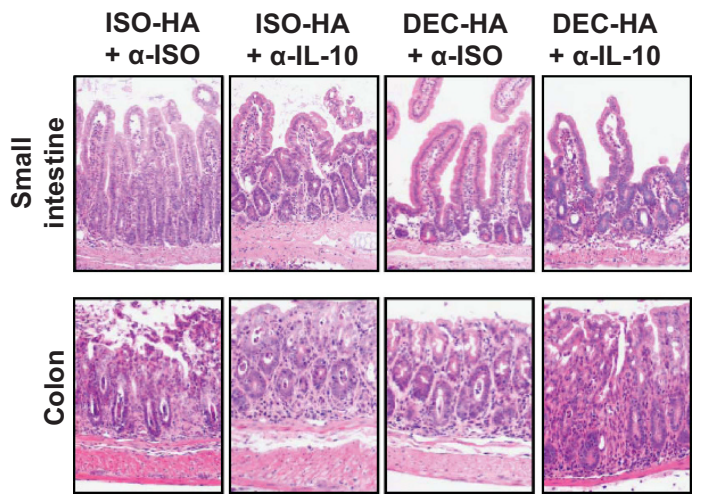

d
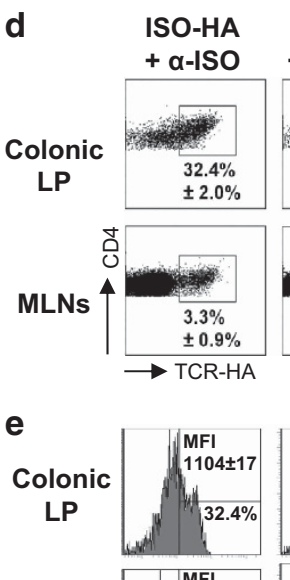

MLNs

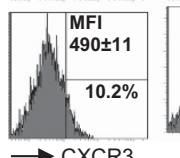

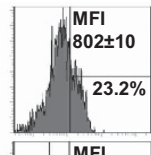

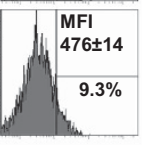

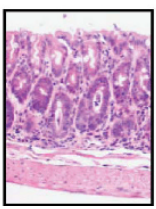

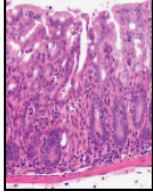

ISO-HA $+\alpha-I L-10$

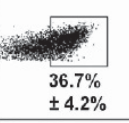

DEC-HA $+\alpha$-ISO

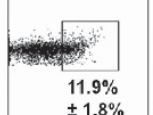

DEC-HA
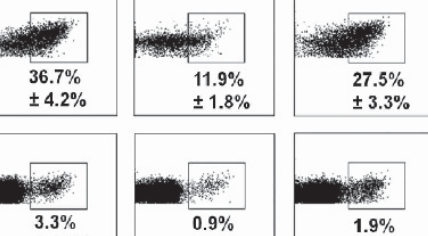

$\pm 1.3 \%$

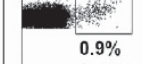

$\pm \mathbf{0 . 3} \%$

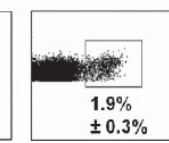
$+\alpha-I L-10$
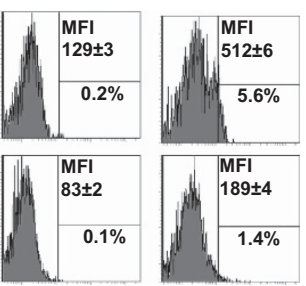

b

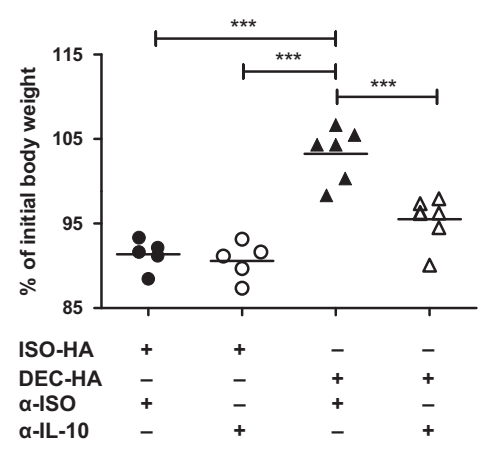

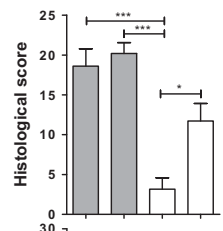
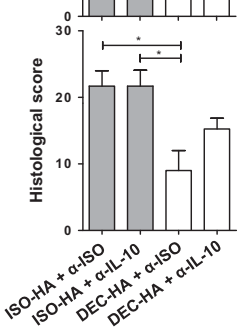

Figure 7 DEC-HA-mediated protection from intestinal inflammation is abolished after interleukin (IL)-10 neutralization. (a) VILLIN-HA transgenic mice were injected intraperitoneally (i.p.) with DEC-HA or ISO-HA on days -2 and -1 . Additionally, mice received daily i.p. injections of a neutralizing $\alpha$-IL-10 antibody or an isotype control antibody ( $\alpha$-ISO) starting on day -2 . On day $0, \mathrm{HA}$-specific Th1 cells were adoptively transferred, and mice were monitored for the loss of body weight and clinical signs of sickness. On day 5 after transfer, (b) the body weight of the mice was determined, and (c) histopathological analysis was performed on sections of the small intestine and colon. (d) Frequencies and (e) C-X-C chemokine receptor 3 surface expression of $\mathrm{HA}$-specific CD4 ${ }^{+} \mathrm{T}$ cells in the colonic lamina propria (LP) and mesenteric lymph nodes (MLNs) of transferred mice were determined by flow cytometry. Data from two independent experiments are shown as means \pm s.e.m. Statistical analysis was performed with one-way analysis of variance $\left({ }^{\star} P<0.05\right.$; $\left.{ }^{* * *} P<0.001\right)$. MFI, mean fluorescence intensity.

initial T-cell transfer, DEC-HA or ISO-HA were injected i.p. followed by a second transfer of HA-specific Th1 cells to induce an inflammatory relapse. Strikingly, DEC-HA-treated VILLINHA mice showed no loss of body weight (Figure 8g) and significantly less inflammation of the gut compared with ISO-HA-treated control mice (Figure $\mathbf{8 h}$ ). These results clearly indicate that DEC-205-mediated antigen targeting could be utilized as a potential IBD therapy.

\section{DISCUSSION}

IBD is characterized by chronic, uncontrolled inflammation in the intestinal mucosa. Although the etiology is poorly 
understood, it is widely accepted that an inappropriate immune response toward otherwise harmless commensal bacteria is a crucial part of the pathogenesis. Chemokines contribute to the inflammatory process through their involvement in cellular trafficking of effector cells. In fact, CXCR3 and its corresponding ligands have been shown to be differentially expressed at sites of colitis in mice and in IBD patients. ${ }^{21-23}$ We found that the percentage of CXCR3-expressing CD4 ${ }^{+}$ $\mathrm{T}$ cells was significantly lower in PBMCs from patients with active CD or UC and exhibited a gradual decrease from healthy control subjects to patients with disease in remission to patients with active disease. In contrast, in healthy volunteers and in patients with IBD in remission $\mathrm{CD} 4{ }^{+} \mathrm{CXCR} 3{ }^{+} \mathrm{T}$ cells were only barely detectable in the colon and ileum, but their numbers were significantly elevated during active $\mathrm{CD}$ or UC. These findings suggest an enhanced migration of $\mathrm{CD}^{+}{ }^{+} \mathrm{CXCR}^{+}{ }^{+}$effector $\mathrm{T}$ cells during inflammation of the gut and imply that CXCR3 could be a suitable target for treating IBD.

In the IL-10 ${ }^{-1-}$ colitis mouse model, CXCL10 and CXCR3 are highly expressed in the gut, and the application of an $\alpha$ CXCL10 antibody attenuates the severity of colitis. ${ }^{21}$ Currently, clinical trials using CXCL10 antibody (MDX-1100) are demonstrating some promise for treatment of patients with UC or rheumatoid arthritis. ${ }^{24}$ In addition, simultaneous blocking of CCR2, CCR5, and CXCR3 by TAK799, a nonpeptide inhibitor, was shown to result in similar disease mitigation in mice. ${ }^{22}$ In the present study, we found that the systemic blocking of CXCR3 by an antagonist resulted in markedly attenuated disease severity. In line with our results, dextran sodium sulfate-induced colitis was attenuated in $\mathrm{CXCR}^{-1-}$ mice because of impaired cellular trafficking to the gut. $^{25}$

However, none of these therapies is disease or antigen specific, and all of them generally target events downstream of the inflammatory cascade. The real challenge is to develop more specific approaches for preventing the initiation and perpetuation of the inflammatory cascade before tissue injury occurs. One approach could involve the induction or re-establishment of immunological tolerance against disease-specific antigens. It has been previously shown that targeting antigen to DEC-205 on immature DCs leads to highly effective immunological tolerance by inducing antigen-specific Foxp $3^{+}$Tregs when this process occurs in the steady state. ${ }^{13,14}$ We show here for the first time that targeting antigen to DEC-205 in the context of Th1 cell responses downregulates the expression of CXCR3 on antigen-specific Th1 cells and interferes with the migration of these cells to the gut. Strikingly, targeting antigen to DEC-205 was even more effective as a therapeutic strategy in preventing antigen-specific gut inflammation than was systemic treatment with a CXCR3 antagonist. Moreover, this approach successfully protected mice in the remission phase from an inflammatory relapse. This finding underlines the need for more precise and antigen-specific approaches to treatment of IBD and shows the potential benefit of DEC-205-mediated antigen delivery in human IBD.
For IBD, great efforts have been made in trying to identify an antigen that acts as a target of local immune responses, but the final antigen or antigen composition is not yet identified. However, CBir1, a bacterial flagellin, was discovered by serological expression cloning from spontaneously colitic $\mathrm{C} 3 \mathrm{H} / \mathrm{HeJBir}$ mice. $^{26}$ This is the first antigen to be linked between mice and humans and has been shown to have a direct pathogenic effect on the development of T-cell-mediated colitis in mice. ${ }^{26,27}$ In addition, LP mononuclear cells isolated from CD patients produce more IFN- $\gamma$ after stimulation with CBirl flagellin than do LP mononuclear cells from healthy control subjects, a finding supporting a pathogenic role for CBir1-specific responses in $\mathrm{CD} .{ }^{28}$ Intriguingly, CBir1 flagellin antibody reactivity marks a subset of patients with a complicated course of CD. ${ }^{29,30}$ The broad recognition of this flagellin in various mouse models and in humans with CD indicates that CBir1 is among the immunodominant antigens of the microbiota in IBD. In this context, CBir 1 might be an interesting target for antigen-directed therapy of the disease. Therefore, it is of great interest whether targeting CBir 1 to DEC-205 could be sufficient to induce CBir1-specific tolerance or to modulate CBir1specific Th1 responses in vitro and in vivo.

IL-10 is expressed by many cell types of the innate and adaptive immune systems and is directly involved in the control of intestinal inflammation. ${ }^{31,32}$ In this study, we found that the secretion of IL-10 by DCs was enhanced after antigen targeting to DEC-205. We demonstrated that in vivo blocking of IL-10 abrogates the therapeutic effect of targeting antigen to DEC-205. Because CD $4{ }^{+}$CXCR $3{ }^{+}$Th1 cells weakly express IL-10R, it is likely that IL-10 indirectly controls adaptive immune responses by signaling on antigen-presenting cells. In particular, it was demonstrated that IL-10 inhibits the differentiation and proliferation of Th1 cells by selectively inhibiting the function of antigen-presenting cells. ${ }^{33,34}$ In accordance with this, stimulation of Th1 cells in the presence of IL-10 in vitro does not alter CXCR3 expression. Therefore, DC-derived IL-10 may be involved in the maintenance of steady-state DCs by an autocrine feedback loop. Of note, higher IL-10 expression induced by an $\alpha$-DEC-205 antibody alone or coupled to an irrelevant antigen does not protect from experimental colitis (see Supplementary Figure S5), demonstrating that only the combination of antigen-specific TCR stimulation and enhanced IL-10 production is sufficient to modulate the migration behavior of effector cells. These findings clearly suggest a novel mechanism by which IL-10 can act indirectly on effector T-cell phenotype and function and orchestrate intestinal immune responses. However, we cannot exclude that other mechanisms may have an important role in maintaining tolerance in the used inflammation model. Neither treatment with CXCR3 antagonist nor the use of a neutralizing $\alpha$-IL-10 antibody had the same protective effect as it was seen by treatment with DEC-HA. Of note, apart from IL-10 also the expression of TGF- $\beta$ was increased in DCs of the spleen and MLNs of DEC-HA-treated mice (see Supplementary Figure S4a). The anti-inflammatory role of TGF- $\beta$ especially in the gut mucosa and its properties to induce 
Foxp $3^{+}$Tregs has been described previously. ${ }^{35,36}$ Although Foxp $3^{+}$Tregs were not affected by DEC-HA treatment, the possibility remains that elevated TGF- $\beta$ levels are involved in the observed protection. However, adoptive co-transfer of
DEC-HA-treated CD4 ${ }^{+}$T cells with freshly polarized HAspecific Th1 cells into naive recipient mice did not protect from severe intestinal inflammation (see Supplementary Figure S7). We therefore conclude that dominant tolerance does not have a

a

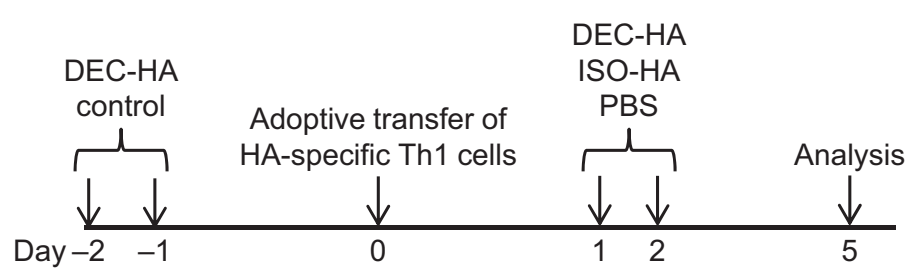

b

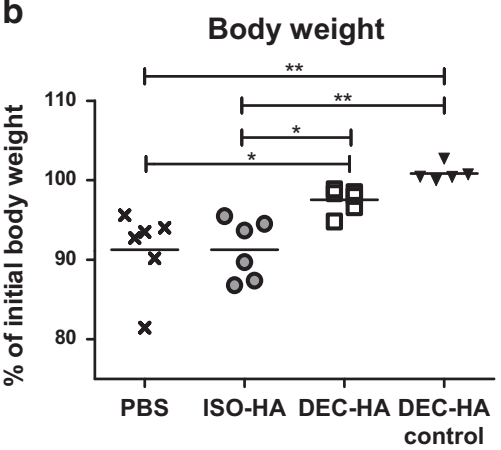

d

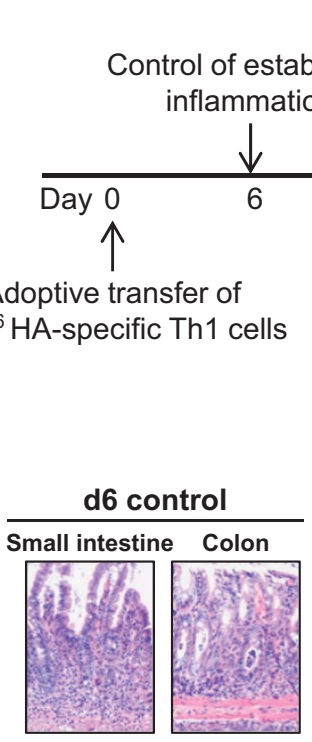

g

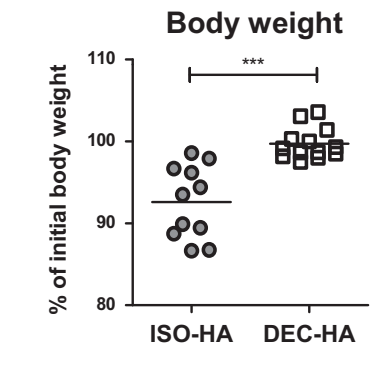

f
C
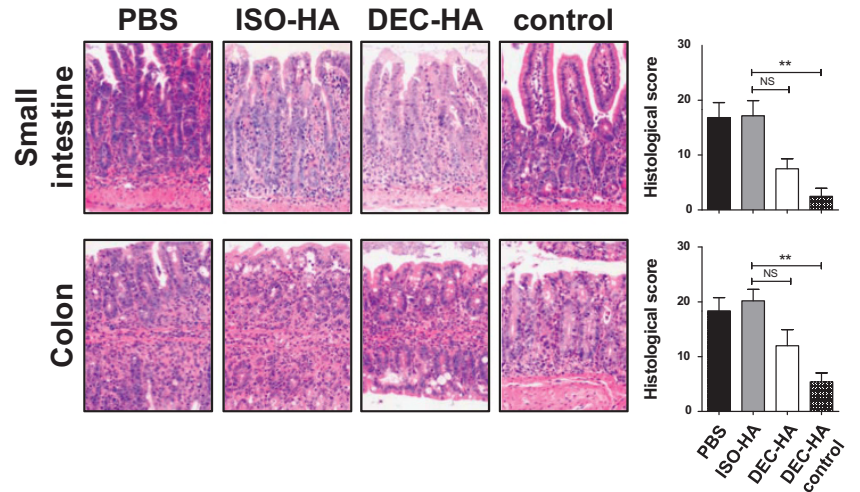

e

Body weight during recovery

ISO-HA
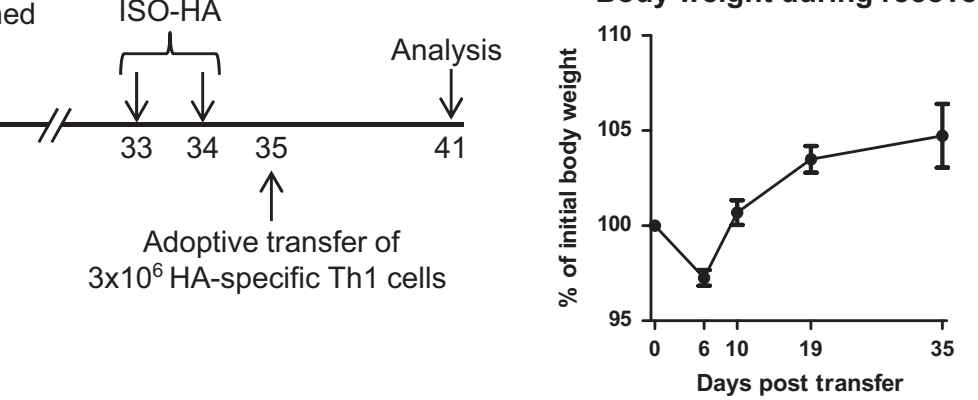

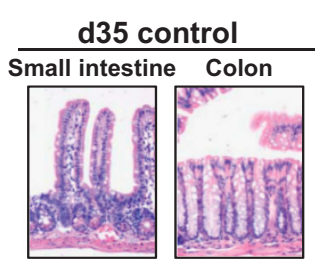

h
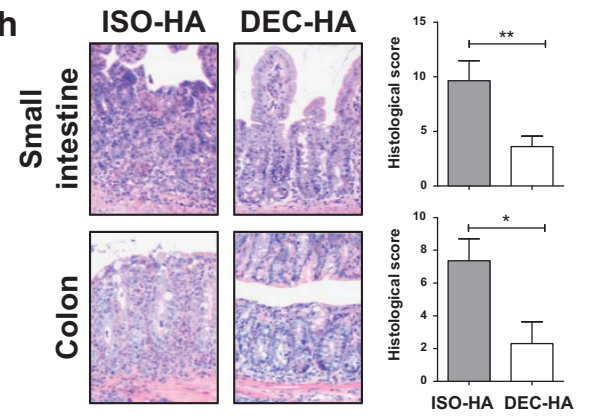
crucial role in the protective activity seen after DEC-HA treatment in our inflammation model.

In summary, our findings show that targeting antigen to DEC-205 interferes with the function of Th1 cells by activating immunomodulatory mechanisms. Targeting DEC-205 with an antibody-antigen complex leads to enhanced secretion of IL-10 and this in turn modulates the ability of $\mathrm{CD} 4{ }^{+} \mathrm{CXCR} 3{ }^{+} \mathrm{T}$ cells to migrate to sites of inflammation by downregulating CXCR3 expression. Together, these mechanisms can prevent autoimmune-mediated inflammation in the small intestine and colon. Because it has been demonstrated that human DCs express a homologous DEC-205 molecule on their surface ${ }^{37}$ and that the numbers of $\mathrm{CD}^{+}{ }^{+} \mathrm{CXCR} 3^{+}$effector $\mathrm{T}$ cells are increased in the colon and ileum during inflammation but not during remission, future investigation may be initiated to elucidate the potential benefits of targeting DEC-205 in human IBD.

\section{METHODS}

Human samples. Human samples were acquired from CD and UC patients. For collection of PBMCs, blood was drawn from patients with disease in remission or with active disease; healthy volunteers served as control subjects (active $\mathrm{CD}, n=3$; $\mathrm{CD}$ in remission, $n=3$; active $\mathrm{UC}$, $n=4$; UC in remission, $n=13$; healthy control subjects, $n=10$ ). For immunofluorescence analysis, mucosal biopsy samples were obtained from subjects undergoing ileocolonoscopy: subjects with active CD, ileum, $n=5$; subjects with $C D$ in remission, ileum, $n=3$; subjects with active $\mathrm{CD}$, colon, $n=5$; subjects with $\mathrm{CD}$ in remission, colon, $n=3$; subjects with active UC, colon, $n=5$; and subjects with UC in remission, colon, $n=3$. Biopsy samples were taken from the terminal ileum of CD patients and control subjects. Colonic tissue was obtained from CD patients, UC patients, and control subjects. CD- or UCspecific tissue from inflamed and non-inflamed mucosa during remission of $\mathrm{CD}$ was confirmed by histopathological analysis. Patients undergoing ileocolonoscopy for gastrointestinal bleeding, carcinoma screening, or abdominal pain were used as control subjects (ileum, $n=5$; colon, $n=5$ ). For these patients, tissue was taken from macroscopically healthy tissue, and histological analysis of biopsy samples indicated normal tissue.

The diagnosis of CD or UC was established by standard criteria. Treatment for CD and UC consisted of mesalamine, budesonide, prednisolone, thiopurines, and methotrexate. Control subjects had not received immunomodulatory drugs. Experiments were approved by the local authorities (Medical Faculty, University of Lübeck: 02-073, 03-043). Informed consent was obtained from all patients.

\section{Immunofluorescence microscopy. See Supplementary Methods.}

Mice. BALB/c mice were obtained from Harlan Winkelmann (Borchen, Germany). VILLIN-HA transgenic mice express A/PR/34 influenza HA under the enterocyte-specific villin promoter. $^{38}$
TCR-HA transgenic mice harbor $\mathrm{CD}^{+} \mathrm{T}$ cells expressing an $\alpha / \beta$-TCR specific for the MHC class II $\mathrm{H}_{2} \mathrm{E}^{\mathrm{d}}: \mathrm{HA}_{110-120}$-restricted epitope of the HA protein. ${ }^{39}$ IL-10 $0^{\text {flox/flox }}$ mice were described elsewhere ${ }^{40}$ and kindly provided by Axel Roers. CD11c-cre mice were described by Canton et al. ${ }^{41} \mathrm{CD} 11 \mathrm{c}$-cre and C57BL/6 mice were obtained from Jackson (Sulzfeld, Germany). OT-II transgenic mice harbor $\mathrm{CD}^{+}{ }^{+} \mathrm{T}$ cells expressing an $\alpha / \beta$-TCR specific for the MHC class II-restricted epitope of the OVA-protein $\left(\mathrm{OVA}_{323-339}\right) .{ }^{42}$ All animal experiments were performed in accordance with institutional, state, and federal guidelines (approved by the Landesamt für Natur, Umwelt und Verbraucherschutz North Rhine-Westphalia, Germany).

\section{Conjugation of antigens to an $\alpha$-DEC-205 monoclonal antibody.} Conjugation of the $\mathrm{HA}_{110-120}$ peptide or the $\mathrm{OVA}_{323-339}$ peptide to the DEC-205-specific antibody was performed as previously described. ${ }^{13}$ In brief, $\alpha$-DEC-205 monoclonal antibody (NLDC-145) or an IgG control antibody (GL117) was purified from hybridoma supernatants and coupled to activated $\mathrm{HA}_{110-120}$ or activated $\mathrm{OVA}_{323-}$ ${ }_{339}$ peptide with the heterobifunctional crosslinker succinimidyl $4-[\mathrm{N}$ maleimidomethyl] cyclohexane-1-car-boxylate (Pierce, Rockford, IL) according to the manufacturer's protocol (DEC-HA/ISO-HA and DEC-OVA/ISO-OVA, respectively).

Antibodies and flow cytometry. See Supplementary Methods.

RNA isolation and quantitative real-time PCR. See Supplementary Methods.

Isolation and flow cytometric cell sorting of lymphocytes. Spleens and MLNs were mashed through $70-\mu \mathrm{m}$ cell strainers and washed with erythrocyte lysis buffer or PBS containing $2 \%$ fetal calf serum and $2 \mathrm{~mm}$ EDTA, respectively. Lymphocytes from the LP of colon were isolated as described previously. ${ }^{38}$ In brief, colons were washed with ice-cold PBS and cut into small pieces; the pieces were washed in PBS supplemented with $2 \mathrm{~mm}$ EDTA and cell culture media under constant stirring. Colons then underwent digestion with collagenase IV (SigmaAldrich, Bonn, Germany) for $90 \mathrm{~min}$ at $37^{\circ} \mathrm{C}$. Single-cell suspensions were obtained by passing suspensions through $70-\mu \mathrm{m}$ cell strainers. $\mathrm{CD} 4{ }^{+} \mathrm{T}$ cells were enriched from the spleens of TCR-HA or OT-II mice with autoMACS technology (Miltenyi Biotec, Bergisch-Gladbach, Germany) according to the manufacturer's instructions. Pure populations of $\mathrm{HA}$-specific CD4 ${ }^{+} \mathrm{T}$ cells were obtained by staining cells with $\alpha$-CD4 and an antibody against the HA-specific TCR and sorting them by flow cytometry on an ARIA II sorter (BD Bioscience, Heidelberg, Germany). Pure populations of OVA-specific CD ${ }^{+}$ $\mathrm{T}$ cells were obtained by staining cells with $\alpha$-CD 4 and an antibody against the TCR V $\beta 5$ chain followed by flow cytometric sorting. Human PBMCs were obtained by diluting freshly isolated blood 1:1 with sterile PBS and then isolating PBMCs with density gradient centrifugation using the Lymphoprep gradient technique (Stemcell, Cologne, Germany).

Isolation and stimulation of DCs. Spleens were cut into small pieces and were then treated with $1 \mathrm{mg} \mathrm{ml}^{-1}$ collagenase type D (Roche, Mannheim, Germany) and $10 \mu \mathrm{g} \mathrm{ml}^{-1}$ DNase I type II (SigmaAldrich) diluted in PBS with $2 \%$ fetal calf serum and 2 mM EDTA for $45 \mathrm{~min}$ at $37^{\circ} \mathrm{C}$. The remaining tissue was mechanically minced and

Figure 8 DEC-HA treatment protects VILLIN-HA mice from intestinal inflammation after inflammatory relapse in the gut. HA-specific T helper type 1 (Th1) cells were adoptively transferred into VILLIN-HA transgenic mice. (a) On days 1 and 2 after transfer, mice were injected intraperitoneally (i.p.) with DEC-HA, ISO-HA, or phosphate-buffered saline (PBS). As a control, one group of mice was i.p. injected with DEC-HA on days -1 and -2 prior to transfer (DEC-HA control). On day 5 after transfer, (b) body weight and (c) histopathological analysis of the small intestine and colon was performed. HA-specific Th1 cells were adoptively transferred into VILLIN-HA mice for the induction of intestinal inflammation. On days 33 and 34 , VILLIN-HA mice were injected i.p. with ISO-HA or DEC-HA followed by another adoptive transfer of HA-specific Th1 cells. (d) On day 41, mice were analyzed. (e) The body weight was monitored throughout the recovery phase. (f) On days 6 and 35 after transfer, control mice were analyzed for histopathological signs of inflammation. (g) Body weight and (h) histopathological analysis of colon and small intestine of ISO-HA and DEC-HA treated mice on day 41 are depicted. Data from two independent experiments are shown as means \pm s.e.m. Statistical analysis was performed with one-way analysis of variance $\left({ }^{\star} P<0.05\right.$; ${ }^{\star \star} P<0.01 ;{ }^{\star \star \star} P<0.001$; NS, not significant). 
filtered through a $40-\mu \mathrm{m}$ cell strainer. Cells were washed in PBS containing $2 \%$ fetal calf serum and $2 \mathrm{~mm}$ EDTA. CD11 $\mathrm{c}^{+}$cells were positively selected with autoMACS technology according to the manufacturer's instructions (Miltenyi Biotec). DCs were stimulated overnight with $100 \mathrm{ng} \mathrm{ml}^{-1}$ lipopolysaccharide (Sigma-Aldrich). Cytokines in cell culture supernatants were quantified with the Procarta Cytokine Assay Kit (Panomics, Santa Clara, CA) according to the manufacturer's instructions. The assay was run with a Luminex 200 system using the Luminex IS software (Luminex Corporation, Austin, TX).

Th1 cell polarization. HA-specific $\mathrm{CD} 4{ }^{+} \mathrm{T}$ cells were sorted by flow cytometry and activated in vitro with plate-bound $\alpha$-CD3 $\left(5 \mu \mathrm{g} \mathrm{ml}^{-1}\right)$ and soluble $\alpha$-CD28 $\left(1 \mu \mathrm{g} \mathrm{ml}^{-1}\right)$ (both BD Bioscience) and were additionally polarized into IFN- $\gamma$-producing Th1 cells by adding recombinant mouse IL-12 (20 $\mathrm{ng} \mathrm{ml}^{-1}$ ) (R\&D Systems, Wiesbaden, Germany) and $\alpha$-IL-4 antibody $\left(200 \mathrm{ng} \mathrm{ml}^{-1}\right.$ ) (eBioscience, San Diego, CA) to the cell culture media. At day 6, successful Th1 cell polarization was measured by FACS staining of the cells for IFN- $\gamma$. For the generation of OVA-specific Th1 cells, OVA-specific CD4 ${ }^{+}$T cells were sorted from the spleens of OT-II mice and polarized in vitro as mentioned above.

Induction of intestinal inflammation in VILLIN-HA transgenic mice. $3 \times 10^{6}$ HA-specific Th1 cells were adoptively transferred into each VILLIN-HA transgenic mouse. Mice were monitored daily for signs of sickness (i.e., loss of body weight) and were killed on day 5 after transfer for analysis.

Treatment of mice with different compounds. VILLIN-HA transgenic mice or wild-type BALB/c were given i.p. injections of $1 \mu \mathrm{g}$ DEC$\mathrm{HA}$, ISO-HA, or $200 \mu \mathrm{l}$ PBS on days -2 and -1 before adoptive transfer of HA-specific Th1 cells. To analyze the role of CXCR3 in the intestinal inflammation model, each VILLIN-HA transgenic mouse was given daily i.p. injections of $150 \mu \mathrm{g}$ of the CXCR3 antagonist Axon 1800 (Axon Medchem, Groningen, The Netherlands) starting on the day of the Th1 cell transfer. In some experiments, each VILLIN-HA transgenic mouse was given daily i.p. injections of $50 \mu \mathrm{g}$ of a neutralizing antibody against IL-10 (clone JES5-2A5) or an isotype control antibody (clone HRPN) (both BioXCell, West Lebanon, NH) from the day of treatment with DEC-HA until 1 day before analysis.

DNA microarray hybridization and analysis. See Supplementary Methods.

\section{Histopathological analysis. See Supplementary Methods.}

Migration assays. HA-specific $\mathrm{CD}^{+} \mathrm{T}$ cells were polarized as described above and were adoptively transferred into BALB/c, CD11ccre $\times$ IL- $10^{\text {flox/flox }}$ or IL- $10^{\text {flox/flox }}$ mice treated with DEC-HA, ISO-HA, or PBS. For mice with the C57BL/6 background, OVA-specific Th1 cells were adoptively transferred into DEC-OVA- or ISO-OVAtreated wild-type mice. On day 6 or 7 after transfer, HA-specific or OVA-specific $\mathrm{CD}_{4}^{+} \mathrm{T}$ cells were re-sorted from the spleens of the respective mice and were subjected to in vitro migration assays using transwell chambers with $5 \mu \mathrm{m}$ polycarbonate filters (Corning, Tewskbury, MA). Therefore, $200 \mathrm{ng} \mathrm{ml}^{-1}$ recombinant mouse CXCL10 (R\&D Systems) was diluted in cell culture media that was added to the lower chamber of the transwell plates. The transwell inserts were placed on top, and $5 \times 10^{5}$ re-sorted HA-specific or OVA-specific $T$ cells were added to the upper chamber, respectively. The plates were then incubated for $4 \mathrm{~h}$ at $37^{\circ} \mathrm{C}$, after which the cells that had migrated into the bottom chamber were collected and counted. The migration index was determined as the ratio of migrated cells toward CXCL10-containing media to cells in the media alone.

Statistical analysis. One-way analysis of variance followed by Bonferroni's multiple comparisons test or Student's $t$-test was used to determine statistical significance, which was set at a level of $P<0.05$.
SUPPLEMENTARY MATERIAL is linked to the online version of the paper at http://www.nature.com/mi

\section{ACKNOWLEDGMENTS}

We thank Heidi Schlichting, Patrick Juszczak, Mechthild Hemmler-Roloff, and Witold Bartosik for excellent technical assistance. This study was supported by grants from the DFG. to A.M.W. and from the Deutsche Krebshilfe to A.M.W. and J. Buer.

\section{AUTHOR CONTRIBUTIONS}

M.W. designed, planned and performed the experiments, analyzed data, and wrote the paper. R.K., A.A., A.F., and E.P. performed experiments and analyzed data. R.G. performed the microarray analysis. K.M., W.H., K.S.L., and J. Buer were involved in the data discussion and in drafting the manuscript. J. Büning initiated the study, provided samples, and discussed data. A.M.W. initiated, organized, and designed the study, wrote the paper, and completed the manuscript.

\section{DISCLOSURE}

The authors declared no conflict of interest.

c) 2016 Society for Mucosal Immunology

\section{REFERENCES}

1. Buckner, J.H. Mechanisms of impaired regulation by CD4(+)CD25(+) FOXP3 $(+)$ regulatory T cells in human autoimmune diseases. Nat. Rev. Immunol. 10, 849-859 (2010).

2. Hanauer, S.B. et al. Human anti-tumor necrosis factor monoclonal antibody (adalimumab) in Crohn's disease: the CLASSIC-I trial. Gastroenterology 130, 323-333 (2006).

3. Lichtenstein, G.R. Emerging prognostic markers to determine Crohn's disease natural history and improve management strategies: a review of recent literature. Gastroenterol. Hepatol. (NY) 6, 99-107 (2010).

4. Dunkin, D., Mehandru, S. \& Colombel, J.F. Immune cell therapy in IBD. Dig. Dis. 32 (Suppl 1), 61-66 (2014).

5. Westendorf, A.M., Fleissner, D., Hansen, W. \& Buer, J. T cells, dendritic cells and epithelial cells in intestinal homeostasis. Int. J. Med. Microbiol. 300, 11-18 (2010).

6. Steinman, R.M., Hawiger, D. \& Nussenzweig, M.C. Tolerogenic dendritic cells. Annu. Rev. Immunol. 21, 685-711 (2003).

7. Bonifaz, L. et al. Efficient targeting of protein antigen to the dendritic cell receptor DEC-205 in the steady state leads to antigen presentation on major histocompatibility complex class I products and peripheral CD8 + T cell tolerance. J. Exp. Med. 196, 1627-1638 (2002).

8. Mahnke, K. et al. The dendritic cell receptor for endocytosis, DEC-205, can recycle and enhance antigen presentation via major histocompatibility complex class II-positive lysosomal compartments. J. Cell Biol. 151, 673-684 (2000).

9. Ettinger, M. et al. Targeting of the hNC16A collagen domain to dendritic cells induces tolerance to human type XVII collagen. Exp. Dermatol. 21, 395-398 (2012).

10. Kretschmer, K. et al. Inducing and expanding regulatory T cell populations by foreign antigen. Nat. Immunol. 6, 1219-1227 (2005).

11. Mukhopadhaya, A. et al. Selective delivery of beta cell antigen to dendritic cells in vivo leads to deletion and tolerance of autoreactive CD8 + Tcells in NOD mice. Proc. Natl. Acad. Sci. USA 105, 6374-6379 (2008).

12. Spiering, R. et al. DEC205 + dendritic cell-targeted tolerogenic vaccination promotes immune tolerance in experimental autoimmune arthritis. $J$. Immunol. 194, 4804-4813 (2015).

13. Mahnke, K., Qian, Y., Knop, J. \& Enk, A.H. Induction of CD4 + /CD25 + regulatory Tcells by targeting of antigens to immature dendritic cells. Blood 101, 4862-4869 (2003).

14. Bruder, D. et al. On the edge of autoimmunity: T-cell stimulation by steady-state dendritic cells prevents autoimmune diabetes. Diabetes 54 , 3395-3401 (2005).

15. Petzold, C., Riewaldt, J., Koenig, T., Schallenberg, S. \& Kretschmer, K. Dendritic cell-targeted pancreatic beta-cell antigen leads to conversion of self-reactive $\mathrm{CD} 4(+) \mathrm{T}$ cells into regulatory $\mathrm{T}$ cells and promotes immunotolerance in NOD mice. Rev. Diabet. Stud. 7, 47-61 (2010). 
16. Stern, J.N. et al. Promoting tolerance to proteolipid protein-induced experimental autoimmune encephalomyelitis through targeting dendritic cells. Proc. Natl. Acad. Sci. USA 107, 17280-17285 (2010).

17. Ring, S., Maas, M., Nettelbeck, D.M., Enk, A.H. \& Mahnke, K. Targeting of autoantigens to DEC205(+) dendritic cells in vivo suppresses experimental allergic encephalomyelitis in mice. J. Immunol. 191, 2938-2947 (2013).

18. Matsuoka, K. et al. T-bet upregulation and subsequent interleukin 12 stimulation are essential for induction of Th1 mediated immunopathology in Crohn's disease. Gut 53, 1303-1308 (2004).

19. Neurath, M.F. et al. The transcription factor T-bet regulates mucosal T cell activation in experimental colitis and Crohn's disease. J. Exp. Med. 195, 1129-1143 (2002).

20. Chaudhry, A. et al. Interleukin-10 signaling in regulatory T cells is required for suppression of Th17 cell-mediated inflammation. Immunity 34, 566-578 (2011).

21. Singh, U.P., Singh, S., Taub, D.D. \& Lillard, J.W. Jr. Inhibition of IFNgamma-inducible protein-10 abrogates colitis in IL-10-/- mice. J. Immunol. 171, 1401-1406 (2003).

22. Tokuyama, H. et al. The simultaneous blockade of chemokine receptors CCR2, CCR5 and CXCR3 by a non-peptide chemokine receptor antagonist protects mice from dextran sodium sulfate-mediated colitis. Int. Immunol. 17, 1023-1034 (2005).

23. Yuan, Y.H. et al. Chemokine receptor CXCR3 expression in inflammatory bowel disease. Inflamm. Bowel Dis. 7, 281-286 (2001).

24. Yellin, M. et al. A phase II, randomized, double-blind, placebo-controlled study evaluating the efficacy and safety of MDX-1100, a fully human antiCXCL10 monoclonal antibody, in combination with methotrexate in patients with rheumatoid arthritis. Arthritis Rheum. 64, 1730-1739 (2012).

25. Chami, B., Yeung, A.W., van, V.C., King, N.J. \& Bao, S. The role of CXCR3 in DSS-induced colitis. PLoS One 9, e101622 (2014).

26. Lodes, M.J. et al. Bacterial flagellin is a dominant antigen in Crohn disease. J. Clin. Invest. 113, 1296-1306 (2004).

27. Elson, C.O. Commensal bacteria as targets in Crohn's disease. Gastroenterology 119, 254-257 (2000).

28. Shen, C., Landers, C.J., Derkowski, C., Elson, C.O. \& Targan, S.R. Enhanced CBir1-specific innate and adaptive immune responses in Crohn's disease. Inflamm. Bowel Dis. 14, 1641-1651 (2008).

29. Papadakis, K.A. et al. Anti-flagellin (CBir1) phenotypic and genetic Crohn's disease associations. Inflamm. Bowel Dis. 13, 524-530 (2007).
30. Targan, S.R. et al. Antibodies to CBir1 flagellin define a unique response that is associated independently with complicated Crohn's disease. Gastroenterology 128, 2020-2028 (2005).

31. Berg, D.J. et al. Enterocolitis and colon cancer in interleukin-10-deficient mice are associated with aberrant cytokine production and CD4(+) TH1-like responses. J. Clin. Invest. 98, 1010-1020 (1996).

32. Hayashi, A. et al. A single strain of Clostridium butyricum induces intestinal IL-10-producing macrophages to suppress acute experimental colitis in mice. Cell Host. Microbe 13, 711-722 (2013).

33. Fiorentino, D.F., Zlotnik, A., Mosmann, T.R., Howard, M. \& O'Garra, A. IL-10 inhibits cytokine production by activated macrophages. J. Immunol. 147, 3815-3822 (1991).

34. Fiorentino, D.F. et al. IL-10 acts on the antigen-presenting cell to inhibit cytokine production by Th1 cells. J. Immunol. 146, 3444-3451 (1991).

35. Coombes, J.L. et al. A functionally specialized population of mucosal CD103 + DCs induces Foxp3 + regulatory T cells via a TGF-beta and retinoic acid-dependent mechanism. J. Exp. Med. 204, 1757-1764 (2007).

36. Sun, C.M. et al. Small intestine lamina propria dendritic cells promote de novo generation of Foxp3 T reg cells via retinoic acid. J. Exp. Med. 204, 1775-1785 (2007).

37. Guo, M. et al. A monoclonal antibody to the DEC-205 endocytosis receptor on human dendritic cells. Hum. Immunol. 61, 729-738 (2000).

38. Westendorf, A.M. et al. CD4 + Tcell mediated intestinal immunity: chronic inflammation versus immune regulation. Gut 54, 60-69 (2005).

39. Kirberg, J. et al. Thymic selection of CD8 + single positive cells with a class II major histocompatibility complex-restricted receptor. J. Exp. Med. 180, 25-34 (1994).

40. Roers, A. et al. T cell-specific inactivation of the interleukin 10 gene in mice results in enhanced $\mathrm{T}$ cell responses but normal innate responses to lipopolysaccharide or skin irritation. J. Exp. Med. 200, 1289-1297 (2004).

41. Caton, M.L., Smith-Raska, M.R. \& Reizis, B. Notch-RBP-J signaling controls the homeostasis of CD8- dendritic cells in the spleen. J. Exp. Med. 204, 1653-1664 (2007).

42. Barnden, M.J., Allison, J., Heath, W.R. \& Carbone, F.R. Defective TCR expression in transgenic mice constructed using cDNA-based alpha- and beta-chain genes under the control of heterologous regulatory elements. Immunol. Cell Biol. 76, 34-40 (1998). 